\title{
HIGHER DEGREES OF DISTRIBUTIVITY AND COMPLETENESS IN BOOLEAN ALGEBRAS
}

\author{
BY \\ E. C. SMITH, JR. AND ALFRED TARSKI
}

Introduction( $\left.{ }^{1}\right)$. Let $\alpha$ and $\beta$ be cardinals. A Boolean algebra $A$ is called $\alpha$-complete if the sum of every set of at most $\alpha$ elements of $A$ exists in $A$. An $\alpha$-complete ideal in a Boolean algebra is analogously defined. The class of all $\alpha$-complete Boolean algebras is a subclass of the class of all Boolean algebras, and this subclass becomes smaller as $\alpha$ increases. A Boolean algebra $A$ is called $(\alpha, \beta)$-distributive if the product of the sums of at most $\alpha$ sets $X$, each consisting of at most $\beta$ elements of $A$, is equal to the sum of all possible products, each of which contains precisely one factor from each $X$, provided that the sums and products in question exist.

In this paper we study $\alpha$-complete and $(\alpha, \beta)$-distributive Boolean algebras, restricting ourselves mainly to the case in which $\alpha$ and $\beta$ are infinite. So far only the case in which both $\alpha$ and $\beta$ are the smallest infinite cardinal has been systematically studied. However, a number of results are known which apply to special $\alpha$-complete Boolean algebras, $\alpha$-complete fields of sets. We shall show, for example, that every $(\alpha, 2)$-distributive Boolean algebra $A$ is $(\alpha, \alpha)$-distributive, and, moreover, if $A$ is $\alpha$-complete, then it is also $\left(\alpha, 2^{(\alpha)}\right)$ distributive. Also, if a Boolean algebra $A$ is $2^{(\alpha)}$-complete, then every $\alpha$ complete prime ideal in $A$ is $2^{(\alpha)}$-complete.

This paper is divided into four sections. The first concerns terminology and symbolism and contains the definition and elementary facts concerning $\alpha$-complete Boolean algebras. Section two explores $(\alpha, \beta)$-distributivity, while section three primarily contains some results which permit one to conclude that an ideal in a Boolean algebra is $\alpha$-complete. Some conditions under which a factor algebra is $\alpha$-complete are established in $\$ 4$. Miscellaneous applications to measure theory are included.

1. Terminology and symbolism. We use ordinary set-theoretic notions and symbolism. In particular, $\in, \subseteq$ (or $\supseteq$ ), $\cup, \cap$ and 0 will denote the relations of membership and inclusion, the operations of union and intersec-

Received by the editors February 5, 1956.

(1) The results presented herein which were obtained by E. C. Smith, Jr. constitute an essential portion of his doctoral thesis presented to the Graduate School of Brown University, 1955, and written under the helpful and encouraging guidance of B. Jonsson. This paper was prepared for publication partly during the period when A. Tarski was a fellow of the John Simon Guggenheim Memorial Foundation. The two authors, working independently, obtained two collections of results on the fundamental notions discussed in this paper. On confronting the two collections, they realized that the intersection of the collections was nonempty; this paper contains their union. 
tion, and the empty set, respectively. We shall let $\{x\}$ denote the family whose sole element is $x$. A family of sets will be called disjointed if, for every pair of distinct members $x$ and $y$ of the family, we have $x \cap y=0$. If $\phi$ is an arbitrary condition, we shall let $\{x \mid \phi\}$ denote the set of all $x$ which satisfy $\phi$. We shall write $\left\{x_{i} \mid \phi(i)\right\}$ for $\left\{y \mid\right.$ for some $i, y=x_{i}$ and $\left.\phi(i)\right\}$. For a family $X=\left\{x_{i} \mid i \in I\right\}$, we shall write either $U X$ or $\bigcup_{i \in I} x_{i}$ and $\cap X$ or $\bigcap_{i \in I} x_{i}$ for the union and intersection of the family. We shall let $\left\langle x_{i} \mid i \in I\right\rangle$ denote a system indexed by $I$; that is, the function $f$ with domain $I$ and such that $f(i)=x_{i}$ for $i \in I$. In particular, if $I$ is an ordinal, then this system is called a sequence of type $I$. (Every ordinal $\alpha$ is the set of all smaller ordinals, so that the two statements " $\beta \in \alpha$ " and " $\beta<\alpha$ " are equivalent.) A sequence of type two is simply denoted $\langle x, y\rangle$. For arbitrary sets $A$ and $B$, we shall let $A \times B=\{z \mid$ for some $x \in A$ and some $y \in B, z=\langle x, y\rangle\}$, the usual Cartesian product of $A$ and $B$. Moreover, we shall let $A^{B}$ denote the set of all functions on $B$ and into $A$. Consequently, for ordinals $\alpha$ and $\beta, A^{\alpha \times \beta}$ is what is of ten called a double sequence with range in $A$. Note that for an ordinal $\alpha, 2^{\alpha}$ denotes the set of all functions on $\alpha$ to 2 . A member of the range of $A^{\alpha}$ (or $\left.A^{\alpha \times \beta}\right)$ is often written $a_{\xi}$ instead of $a(\xi)$ (or $a_{\xi, \rho}$ instead of $a(\xi, \rho)$ ).

For the sake of uniformity, we shall make more explicit use of the well ordering theorem than is necessary, even to the extent of defining distributivity in terms of sequences rather than more general sets.

Small Greek letters will denote ordinals. We shall identify cardinals with initial ordinals, and reserve the letters $\alpha$ and $\beta$ to stand for cardinals at least as large as 2. The $\beta$ th cardinal arithmetic power of $\alpha$ will be denoted $\alpha^{(\beta)}$. If $X$ is a set of cardinals, $\sum^{*} X$ or $\sum_{\beta \in X}^{*} \beta$ will denote the least (cardinal) upper bound of $X$. We shall use the symbol $\rho^{*}$ for $\sum_{\xi<\rho}^{*} 2^{(\xi)}$. For any set $A, \kappa(A)$ will stand for the power (cardinal) of $A$. A cardinal $\beta$ is called a limit number if there is no largest cardinal among all those smaller than $\beta$. We say that $\beta$ is a strong limit number if $2^{(\alpha)}<\beta$ whenever $\alpha<\beta$. A cardinal $\beta$ is called singular if it can be represented as the sum of fewer than $\beta$ cardinals, each of which is smaller than $\beta$. All other cardinals are called regular. Every infinite singular cardinal is a limit number. Regular limit numbers are among the so-called inaccessible numbers, sometimes described as those cardinals not attainable (either weakly or strongly) from smaller cardinals. In particular, $\beta$ is said to be strongly attainable from $\alpha$ if there is no regular limit number $\xi$ such that $\alpha<\xi \leqq \beta$. Moreover, $\beta$ is said to be weakly attainable from $\alpha$ if there is no regular, strong limit number $\xi$ such that $\alpha<\xi \leqq \beta\left({ }^{2}\right)$. A necessary and sufficient condition that $\beta$ be a cardinal not weakly attainable from any smaller cardinal is that $\beta^{\left(2^{(a)}\right)}=\beta$ whenever $\alpha<\beta$.

We assume a familiarity with the definitions and basic properties of Boolean algebras $\left({ }^{3}\right)$. We shall not distinguish between a Boolean algebra and the

(2) See [16]. Numbers in brackets refer to the bibliography at the end of the paper.

(3) For a treatment of these ideas, see [1]. 
set of all its elements. We shall use $+, \cdot,-$, and - to denote the Boolean operations of addition (join), multiplication (meet), complementation and subtraction, respectively. In addition, if $\phi$ is a Boolean expression, we shall sometimes write $[\phi]-$ for $\bar{\phi}$. We shall let $\sum$ and $\Pi$ be the usual generalizations of + and $\cdot ;$ that is, if $X=\left\{x_{\xi} \mid \xi<\rho\right\}$ is a subset of a Boolean algebra, we denote its algebraic sum, when it exists, by either $\sum X$ or $\sum_{\xi<p} x_{\xi}$, and similarly for its product. We shall use the symbols 0 and 1 for the zero and unit elements, respectively, of any Boolean algebra as well as for the ordinary numbers zero and one. A subset of a Boolean algebra is called disjointed if $x \cdot y=0$ for every pair of distinct elements $x$ and $y$ of that subset. Moreover, for any subset $X$ of a Boolean algebra, we shall let $\delta(X)$ denote the least cardinal $\rho$ such that the power of every disjointed subset of $X$ is at most $\rho$. It is known (see [4]) that if the power of every disjointed subset of $X$ is less than $\delta(X)$, then $\delta(X)$ is a regular limit number.

If $a$ is an element of a Boolean algebra $A$, then $[a]$ will denote the principal ideal generated by $a$ in $A$; that is, $[a]=\{b \mid b \in A$ and $b \leqq a\}$. We note that $[a]$ may itself be considered as a Boolean algebra.

If $I$ is an ideal in a Boolean algebra $A$, we let $A / I$ be the corresponding factor algebra or algebra of cosets. When $a \in A, a / I$ is the coset corresponding to $a ; X / I$ is similarly defined when $X \subseteq A$.

An element $a$ of a Boolean algebra $A$ is called an atom of $A$ provided that $a \neq 0$ and $a \cdot b=a$ whenever $b \in A$ and $a \cdot b \neq 0$. A Boolean algebra is called atomistic if the sum of the set of its atoms is 1 . This means that if $b$ is a nonzero element of an atomistic Boolean algebra $A$, then there is an atom $a$ of $A$ such that $a \cdot b=a$.

Definition 1.1. A Boolean algebra $A$ is $\alpha$-complete if the sum of every subset of $A$ of power at most $\alpha$ exists in $A$.

An $\boldsymbol{\aleph}_{0}$-complete Boolean algebra is of ten called countably complete.

Definition 1.2. A Boolean algebra is complete if it is $\alpha$-complete for every cardinal $\alpha$.

Every Boolean algebra is $\alpha$-complete for every finite $\alpha$. Moreover, if $\beta$ is a singular cardinal and a Boolean algebra $A$ is $\alpha$-complete for every $\alpha<\beta$, then $A$ is also $\beta$-complete $\left({ }^{4}\right)$. The assumption of singularity here is essential.

Definition 1.3. An ideal I in a Boolean algebra is $\alpha$-complete if the sum of every subset of $I$ of power at most $\alpha$ exists and belongs to $I$.

One should note the duality which exists for Boolean algebras. That is, if "sum" and "product" are interchanged in any Boolean algebraic statement, an equivalent statement can easily be obtained. In an $\alpha$-complete Boolean algebra the product of every subset of power at most $\alpha$ exists, etc.

Definition 1.4. A nonempty family of subsets of a given set $S$ is a field of sets if it is closed under the operations of set-theoretical union and complementation with respect to $S$.

(4) See, for example, [10, Theorem 2.16] for the case of fields of sets. 
A consequence of this definition is that a field of sets is closed under settheoretical intersection.

Definition 1.5. A field $F$ of sets is $\alpha$-complete if the union of every subset of $F$ of power at most $\alpha$ belongs to $F$.

Definition 1.6. A field of sets is complete if it is $\alpha$-complete for every cardinal $\alpha$.

Every $\alpha$-complete field of sets is an $\alpha$-complete Boolean algebra. The converse, however, is not true. In fact, although every Boolean algebra is isomorphic to a field of sets, an $\alpha$-complete Boolean algebra need not be isomorphic to an $\alpha$-complete field of sets. The following three statements $\left({ }^{5}\right)$, however, are equivalent for a Boolean algebra $A$ :

(i) $A$ is isomorphic to a complete field of sets.

(ii) $A$ is isomorphic to the field of all subsets of a set.

(iii) $A$ is complete and atomistic.

We shall return to this topic later.

2. $(\alpha, \beta)$-distributivity. If $A$ is a Boolean algebra, $a \in A^{\beta}$ and $b \in A$, then, no matter how large the cardinal $\beta$, the following distributive conditions always hold: $b \cdot \sum_{\xi<\beta} a_{\xi}=\sum_{\xi<\beta}\left(b \cdot a_{\xi}\right)$ and $b+\prod_{\xi<\beta} a_{\xi}=\prod_{\xi<\beta}\left(b+a_{\xi}\right)$.

Definition 2.1. A Boolean algebra $A$ is $(\alpha, \beta)$-distributive if the following is satisfied: Given any double sequence $a \in A^{\alpha \times \beta}$ such that all the sums $\sum_{\eta<\beta} a_{\xi, \eta}$ for $\xi<\alpha$, their product $\prod_{\xi<\alpha} \sum_{\eta<\beta} a_{\xi, \eta}$, and all the products $\prod_{\xi<\alpha} a_{\xi, f(\xi)}$ for $f \in \beta^{\alpha}$ exist, then the sum $\sum_{f \in \beta^{\alpha}} \prod_{\xi<\alpha} a_{\xi, f(\xi)}$ also exists, and we have

$$
\prod_{\xi<\alpha} \sum_{\eta<\beta} a_{\xi, \eta}=\sum_{f \in \beta} \prod_{\xi<\alpha} a_{\xi, f(\xi)} .
$$

It should be noted that in any Boolean algebra $A$, given a double sequence $a \in A^{\alpha \times \beta}$ with the existential properties of the above definition, we always have

$$
\prod_{\xi<\alpha} \sum_{\eta<\beta} a_{\xi, \eta} \geqq \sum_{f \in \beta} \prod_{\xi<\alpha} a_{\xi, f(\xi)}
$$

when the latter sum exists.

The notion of $(\alpha, \alpha)$-distributivity was defined for the first time in [4].

The following theorem has been communicated to us by Mr. D. Scott.

TheOREM 2.2. Let $A$ be a Boolean algebra. The following statements are equivalent:

(0) If $a \in A^{\alpha \times \beta}$ and $\sum_{\eta<\beta} a_{\xi, \eta}$ for $\xi<\alpha, \prod_{\xi<\alpha} \sum_{\eta<\beta} a_{\xi, \eta}, \prod_{\xi<\alpha} a_{\xi, f(\xi)}$ for $f \in \beta^{\alpha}$, and $\sum_{f \in \beta^{\alpha}} \prod_{\xi<\alpha} a_{\xi, f(\xi)}$ all exist, then $\prod_{\xi<\alpha} \sum_{\eta<\beta} a_{\xi, \eta}=\sum_{f \in \beta^{\alpha}} \prod_{\xi<\alpha} a_{\xi, f(\xi) \text {. }}$

(i) $A$ is $(\alpha, \beta)$-distributive.

(ii) If $a \in A^{\alpha \times \beta}$ and $\sum_{\eta<\beta} a_{\xi, \eta}$ for $\xi<\alpha$ and $\prod_{\xi<\alpha} a_{\xi, f(\xi)}$ for $f \in \beta^{\alpha}$ exist, then either both $\prod_{\xi<\alpha} \sum_{\eta<\beta} a_{\xi, \eta}$ and $\sum_{f \in \beta^{\alpha}} \prod_{\xi<\alpha} a_{\xi, f(\xi)}$ exist and are equal, or else neither exist.

(5) This was first established by Lindenbaum and Tarski and is given in [14, Theorem 6]. 
(iii) If $a \in A^{\alpha \times \beta}, \sum_{\eta<\beta} a_{\xi, \eta}$ exists for $\xi<\alpha$ and $\prod_{\xi<\alpha} \sum_{\eta<\beta} a_{\xi, \eta}$ exists and is not 0 , then there is an $f \in \beta^{\alpha}$ such that $\prod_{\xi<\alpha} a_{\xi, f(\xi)}=0$ is false; i.e., either $\prod_{\xi<\alpha} a_{\xi, f(\xi)}$ does not exist or is not 0 .

(iv) If $a \in A^{\alpha \times \beta}, \sum_{\eta<\beta} a_{\xi, \eta}$ exists for $\xi<\alpha$ and there is $a b \in A$ such that $\sum_{\eta<\beta} a_{\xi, \eta}=b>0$ for $\xi<\alpha$, then there is an $f \in \beta^{\alpha}$ such that $\prod_{\xi<\alpha} a_{\xi, f(\xi)}=0$ is false.

Proof. It is clear that (ii) $\Rightarrow(\mathrm{i}) \Rightarrow(0) \Rightarrow$ (iii) $\Rightarrow$ (iv).

Let us assume that $A$ is a Boolean algebra for which (iv) holds. We shall show that (ii) holds also. Let $d$ be a member of $A^{\alpha \times \beta}$ such that $\sum_{\eta<\beta} d_{\xi, \eta}$ exists for every $\xi<\alpha$ and $\prod_{\xi<\alpha} d_{\xi, f(\xi)}$ exists for every $f \in \beta^{\alpha}$.

Suppose that $\prod_{\xi<\alpha} \sum_{\eta<\beta} d_{\xi, \eta}$ exists. Call this product $c$. In order to show that $\sum_{f \in \beta^{\alpha}} \prod_{\xi<\alpha} d_{\xi, f(\xi)}$ exists and equals $c$, it is sufficient to show that

$$
\prod_{\xi<\alpha} d_{\xi, f(\xi)} \leqq c \text { for every } f \in \beta^{\alpha},
$$

and

(2) if $b \in A, b \leqq c$ and $b \cdot \prod_{\xi<\alpha} d_{\xi, f(\xi)}=0$ for every $\operatorname{step} f \in \beta^{\alpha}$, then $b=0$.

We note that

$$
\prod_{\gamma<\alpha} d_{\gamma, f(\gamma)} \leqq \sum_{\eta<\beta} d_{\xi, \eta}
$$

for every $f \in \beta^{\alpha}$ and $\xi<\alpha$, so that (1) is immediate.

Let $b$ be any member of $A$ such that $b \leqq c$ and $b \cdot \prod_{\xi<\alpha} d_{\xi, f(\xi)}=0$ for every $f \in \beta^{\alpha}$. On letting $a_{\xi, \eta}=b \cdot d_{\xi, \eta}$ for $\xi<\alpha$ and $\eta<\beta$, we define an $a \in A^{\alpha \times \beta}$ such that $\sum_{\eta<\beta} a_{\xi, \eta}=b$ for every $\xi<\alpha$. Then

$$
\prod_{\xi<\alpha} a_{\xi, f(\xi)}=b \cdot \prod_{\xi<\alpha} d_{\xi, f(\xi)}=0
$$

for every $f \in \beta^{\alpha}$. Consequently, (iv) implies that $b=0$, and (2) is established.

A similar argument establishes the desired existence and equality when one assumes the existence of $\sum_{f \in \beta^{\alpha}} \prod_{\xi<\alpha} d_{\xi, f(\xi)}$.

Of course Definition 2.1 and Theorem 2.2 could be stated dually by the use of deMorgan's laws. In particular, the dual of the equality in Definition 2.1 would be

$$
\sum_{\xi<\alpha} \prod_{\eta<\beta} a_{\xi, \eta}=\prod_{f \in \beta} \sum_{\xi<\alpha} a_{\xi, f(\xi) .}
$$

We shall not use this dual form.

A Boolean algebra which is $(\alpha, \alpha)$-distributive for every $\alpha$ is called completely distributive.

THEOREM 2.3. If $2 \leqq \alpha^{\prime} \leqq \alpha$ and $2 \leqq \beta^{\prime} \leqq \beta$, then every $(\alpha, \beta)$-distributive Boolean algebra is $\left(\alpha^{\prime}, \beta^{\prime}\right)$-distributive. 
Proof. By Definition 2.1.

We shall consider the opposite question. That is, if $\alpha^{\prime} \leqq \alpha$ and $\beta^{\prime} \leqq \beta$, under what conditions is an $\left(\alpha^{\prime}, \beta^{\prime}\right)$-distributive Boolean algebra necessarily $(\alpha, \beta)$ distributive? The simplest result of this type is the following: if $\alpha^{\prime}=\beta^{\prime}=2$ and $\alpha$ and $\beta$ are finite, then every $\left(\alpha^{\prime}, \beta^{\prime}\right)$-distributive Boolean algebra is $(\alpha, \beta)$ distributive. The following theorem is a stronger, known result.

ThEOREM 2.4. Every Boolean algebra is $(2, \beta)$-distributive.

The proof and further bibliographical references may be found in [15, p. 211, Theorem 15.20].

Theorem 2.5 $\left(^{(6)}\right.$. Every $(\alpha, 2)$-distributive Boolean algebra is $(\alpha, \alpha)$-distributive.

Proof. Let $A$ be an $(\alpha, 2)$-distributive Boolean algebra. The result is immediate if $\alpha$ is finite, so assume otherwise. Let $a$ be an element of $A^{\alpha \times \alpha}$ such that the sums $\sum_{\eta<\alpha} a_{\xi, \eta}$ for $\xi<\alpha$, their product $\prod_{\xi<\alpha} \sum_{\eta<\alpha} a_{\xi, \eta}$ and the products $\prod_{\xi<\alpha} a_{\xi, f(\xi)}$ for $f \in \alpha^{\alpha}$ exist. It is sufficient to show that if

$$
\prod_{\xi<\alpha} \sum_{\eta<\alpha} a_{\xi, \eta}=b^{\prime}>0
$$

for some element $b^{\prime}$ of $A$, then there exists an $f \in \alpha^{\alpha}$ such that

$$
\prod_{\xi<\alpha} a_{\xi, f(\xi)}>0 .
$$

Choose a univalent function $\phi$ on $\alpha \times \alpha$ onto $\alpha$, and let $b \in A^{\alpha \times 2}$ be the sequence such that $b_{\phi(\xi, \eta), 0}=b^{\prime} \cdot a_{\xi, \eta}$ and $b_{\phi(\xi, \eta), 1}=b^{\prime} \cdot\left[a_{\xi, \eta}\right]$ - whenever $\xi, \eta<\alpha$. Consequently,

$$
b^{\prime}=\prod_{\eta<\alpha}\left(b_{\eta, 0}+b_{\eta, 1}\right)
$$

Then there is a $g^{\prime} \in 2^{\alpha}$ for which

$$
\prod_{\eta<\alpha} b_{\eta, o^{\prime}(\eta)}=0
$$

is false; for otherwise, (1) and (3) imply that

$$
\begin{aligned}
0 & <\prod_{\eta<\alpha}\left(b_{\eta, 0}+b_{\eta, 1}\right) \\
& =\sum_{o \in 2^{\alpha}} \prod_{\eta<\alpha} b_{\eta, \theta(\eta)}=0,
\end{aligned}
$$

since $A$ is $(\alpha, 2)$-distributive. Now choose an element $c$ of $A$ such that $c>0$ and $b_{\eta, o^{\prime}(\eta)} \geqq c$ for every $\eta<\alpha$. Since for each $\xi<\alpha$,

(8) The authors have recently found that R. S. Pierce has independently discovered that every $(\alpha, 2)$-distributive, $\alpha$-complete Boolean algebra is $(\alpha, \alpha)$-distributive. See [6]. 


$$
b^{\prime} \cdot \sum_{\eta<\alpha} a_{\xi, \eta}=b^{\prime},
$$

we have

$$
b^{\prime} \cdot \prod_{\eta<\alpha}\left[a_{\xi, \eta}\right]^{-}=0,
$$

so that there must be an $\eta_{\xi}<\alpha$ and a $\lambda_{\xi}<\alpha$ for which

$$
b_{\eta_{\xi}, \theta^{\prime}\left(\eta_{\xi}\right)}=a_{\xi, \lambda_{\xi}} \cdot b^{\prime} \text {. }
$$

Then if $f$ is the member of $\alpha^{\alpha}$ such that $f(\xi)=\lambda_{\xi}$, we have

$$
\prod_{\xi<\alpha} a_{\xi, f(\xi)} \geqq c,
$$

and (2) follows as desired.

Corollary 2.6. Every $(\alpha, \beta)$-distributive Boolean algebra is $(\alpha, \alpha)$-distributive.

Proof. By Theorems 2.4 and 2.5.

We shall make much use of the following result, which is substantially known $\left({ }^{7}\right)$.

Lemma 2.7. Suppose that $\alpha$ is infinite and that $A$ is a complete, atomistic Boolean algebra such that the power $\beta$ of the set of all atoms of $A$ is at most $2^{(\alpha)}$. Then there exists a sequence $c \in A^{\alpha \times 2}$ such that

(i) $c_{\xi, 0}=\left[c_{\xi, 1}\right]-$ for each $\xi<\alpha$,

(ii) for each atom a of $A$ there is an $f \in 2^{\alpha}$ such that $a=\prod_{\xi<\alpha} c_{\xi, f(\xi)}$,

(iii) for each $f \in 2^{\alpha}$ there is at most one atom a of $A$ such that $a \leqq \prod_{\xi<\alpha} c_{\xi, f(\xi)}$.

Moreover, in the case in which $\beta=2^{(\alpha)}$,

(iv) if $U \subseteq \alpha, \kappa(U)<\alpha$, and $g \in 2^{U}$, then $\prod_{\xi \in U} c_{\xi, g(\xi)}$ is the sum of $2^{(\alpha)}$ distinct atoms of $A$.

Proof. Let $B$ be the set of all atoms of $A$; let $\phi$ be a univalent function on $B$ into $\{Y \mid Y \subseteq \alpha\}$, and if $\beta=2^{(\alpha)}$, require $\phi$ to be onto $\{Y \mid Y \subseteq \alpha\}$. For each $\xi<\alpha$ define

$$
\begin{aligned}
& c_{\xi, 0}=\sum\{a \mid a \in B \text { and } \xi \in \phi(a)\}, \\
& c_{\xi, 1}=\sum\{a \mid a \in B \text { and } \xi \in \phi(a)\} .
\end{aligned}
$$

Then (i) follows immediately.

Let $a$ be an arbitrary member of $B$, and let $f$ be the member of $2^{\alpha}$ such that $f(\xi)=0$ when $\xi \in \phi(a)$ and $f(\xi)=1$ when $\xi \notin \phi(a)$. It then follows from (1) that

$$
a \leqq \prod_{\xi<\alpha} c_{\xi, f(\xi)} \text {. }
$$

Consider any $b \in B$ such that $b \neq a$. Then by the choice of $\phi$, there exists an

$\left({ }^{7}\right)$ For the essence of this result in set-theoretic terms, see [17, Lemma 6]. 
$\eta<\alpha$ satisfying either $\eta \in \phi(a)-\phi(b)$ or $\eta \in \phi(b)-\phi(a)$. Note that $B$ is disjointed. Hence, $b \cdot c_{\eta, f(\eta)}=0$, so that $b \cdot \prod_{\xi<\alpha} c_{\xi, f(\xi)}=0$. Consequently, since $A$ is atomistic, it follows from (2) that

$$
a=\prod_{\xi<\alpha} c_{\xi, f(\xi)}
$$

and (ii) is established.

By means of a similar argument one can obtain (iii).

In order to establish (iv), let $U$ be a subset of $\alpha$ of power $<\alpha$, let $g \in 2^{U}$, and let $F$ be the set of all members $f$ of $2^{\alpha}$ satisfying $f(\xi)=g(\xi)$ for every $\xi \in U$. Since $\kappa(\alpha-U)=\alpha$, we have $\kappa(F)=2^{(\alpha)}=\beta$. Moreover,

$$
\prod_{\xi<\alpha} c_{\xi, f(\xi)} \leqq \prod_{\xi \in U} c_{\xi, \sigma(\xi)} \text { for every } f \in F .
$$

Since we assume that $\phi$ is onto $\{Y \mid Y \subseteq \alpha\}$, it can be seen that for every $f \in F, \prod_{\xi<\alpha} c_{\xi, f(\xi)} \in B$; and if $f_{1} \neq f_{2}$ and $f_{1}, f_{2} \in F$, then

$$
\prod_{\xi<\alpha} c_{\xi, f_{1}(\xi)} \neq \prod_{\xi<\alpha} c_{\xi, f_{2}(\xi)}
$$

Now consider an element $b$ of $B$ such that $b \leqq \prod_{\xi \in U} c_{\xi, o(\xi)}$. By (ii) there is an $f^{\prime} \in 2^{\alpha}$ such that $b=\prod_{\xi<\alpha} c_{\xi, f^{\prime}(\xi)}$. We observe that $c_{\xi, 0} \cdot c_{\xi, 1}=0$ for every $\xi<\alpha$. Consequently, $f^{\prime}(\xi)=g(\xi)$ for every $\xi \in U$. That is, $f^{\prime} \in F$. Therefore,

$$
\sum_{j \in F} \prod_{\xi<\alpha} c_{\xi, f(\xi)}=\prod_{\xi \in U} c_{\xi, \theta(\xi)},
$$

which establishes (iv) and completes the proof.

THEOREM 2.8. Every $(\alpha, \alpha)$-distributive, $2^{(\alpha)}$-complete Boolean algebra is $\left(\alpha, 2^{(\alpha)}\right)$-distributive.

Proof. The result is immediate if $\alpha$ is finite, so assume otherwise. Let $\beta=2^{(\alpha)}$, and choose an arbitrary sequence $a \in A^{\alpha \times \beta}$. Define

$$
\begin{aligned}
& b_{\xi, 0}=a_{\xi, 0}, \text { and } \\
& b_{\xi, \eta}=a_{\xi, \eta} \cdot\left[\sum_{\rho<\eta} a_{\xi, \rho}\right]^{-} \text {for } \xi<\alpha \text { and } 0<\eta<\beta .
\end{aligned}
$$

One easily sees that the set $\left\{x \mid x=b_{\xi, \eta}\right.$ and $\left.\eta<\beta\right\}$ is disjointed for each $\xi<\alpha$, so that $\left\{y \mid y=\sum_{\rho \in V} b_{\xi, \rho}\right.$ and $\left.V \subseteq \beta\right\}$ forms a complete, atomistic Boolean algebra, $A_{\xi}$, where the operations are those of $A$ restricted to this set, and $\sum_{\eta<\beta} b_{\xi, \eta}$ is the unit element. For convenience we shall disregard the possibility that some of the $b_{\xi, \eta}$ may be zero and consider $\left\{x \mid x=b_{\xi, \eta}\right.$ and $\left.\eta<\beta\right\}$ as the set of atoms of $A_{\xi}$. We may apply Lemma 2.7 to these algebras. Thus, for each $\xi<\alpha$ there is a sequence $c^{\xi} \in A^{\alpha \times 2}$ such that

$$
\sum_{\eta<\beta} b_{\xi, \eta}=c_{\rho, 0}^{\xi}+c_{\rho, 1}^{\xi}
$$


for each $\rho<\alpha$; and for each $b_{\xi, \eta}$ (with $\xi<\alpha$ and $\eta<\beta$ ) there is a function $f_{\xi, \eta} \in 2^{\alpha}$ such that

$$
b_{\xi, \eta}=\prod_{\rho<\alpha} c_{\rho, f_{\xi, \eta}(\rho)}^{\xi}
$$

Now choose a univalent function $\phi$ on $\alpha \times \alpha$ onto $\alpha$, and let $c \in A^{\alpha \times 2}$ be the sequence such that

$$
c_{\phi(\xi, \rho), \eta}=c_{\rho, \eta}^{\xi} \text { whenever } \xi<\alpha, \rho<\alpha \text { and } \eta<2 .
$$

By $(\alpha, \alpha)$-distributivity,

$$
\prod_{\lambda<\alpha}\left(c_{\lambda, 0}+c_{\lambda, 1}\right)=\sum_{f \in 2} \prod_{\lambda<\alpha} c_{\lambda, f(\lambda)}
$$

Also, if $g$ is an arbitrary member of $\beta^{\alpha}$,

$$
\begin{aligned}
\prod_{\xi<\alpha} b_{\xi, g(\xi)} & =\prod_{\xi<\alpha} \prod_{\rho<\alpha} c_{\rho, f_{\xi, g(\xi)}(\rho)}^{\xi} \\
& =\prod_{\lambda<\alpha} c_{\lambda, f(\lambda)}
\end{aligned}
$$

for a suitable $f \in 2^{\alpha}$; and every $f \in 2^{\alpha}$ is similarly related to a $g \in \beta^{\alpha}$. Then (2), (3), (4) and (5) imply that

$$
\begin{aligned}
\prod_{\xi<\alpha} \sum_{\eta<\beta} b_{\xi, \eta} & =\prod_{\lambda<\alpha}\left(c_{\lambda, 0}+c_{\lambda, 1}\right) \\
& =\sum_{f \in \in_{2}^{\alpha}} \prod_{\lambda<\alpha} c_{\lambda, f(\lambda)} \\
& =\sum_{o \in \beta^{\alpha}} \prod_{\xi<\alpha} b_{\xi, \mathbf{g}(\xi)} .
\end{aligned}
$$

However, from (1) it follows that if $\xi<\alpha$, then

$$
\sum_{\eta<\beta} a_{\xi, \eta}=\sum_{\eta<\beta} b_{\xi, \eta}
$$

and $b_{\xi, \eta} \leqq a_{\xi, \eta}$ for $\eta<\beta$, so that by (6),

$$
\begin{aligned}
\prod_{\xi<\alpha} \sum_{\eta<\beta} a_{\xi, \eta} & =\prod_{\xi<\alpha} \sum_{\eta<\beta} b_{\xi, \eta} \\
& =\sum_{o \in \beta^{\alpha}} \prod_{\xi<\alpha} b_{\xi, o(\xi)} \\
& \leqq \sum_{o \in \beta^{\alpha}} \prod_{\xi<\alpha} a_{\xi, \sigma(\xi)} .
\end{aligned}
$$

But we know that in any case,

Hence, by (7)

$$
\prod_{\xi<\alpha} \sum_{\eta<\beta} a_{\xi, \eta} \geqq \sum_{o \in \beta^{\alpha}} \prod_{\xi<\alpha} a_{\xi, o(\xi)} .
$$




$$
\prod_{\xi<\alpha} \sum_{\eta<\beta} a_{\xi, \eta}=\sum_{o \in \beta^{\alpha}} \prod_{\xi<\alpha} a_{\xi, \sigma(\xi)}
$$

The above proof can easily be modified to obtain the stronger result that if $\beta \leqq 2^{(\alpha)}$, then any $\beta$-complete, $(\alpha, \alpha)$-distributive Boolean algebra is $(\alpha, \beta)$ distributive. Observe that this is a weak converse of Corollary 2.6.

Theorem 2.9. Suppose that $\beta$ is a singular, strong limit cardinal and that $A$ is a $\beta$-complete Boolean algebra which is $(\alpha, \beta)$-distributive for every $\alpha<\beta$. Then $A$ is $(\beta, \beta)$-distributive.

Proof. By Theorem 2.5 we need only show that $A$ is $(\beta, 2)$-distributive. To this end, let $a \in A^{\beta \times 2}$ be such a sequence that

$$
\prod_{\xi<\beta}\left(a_{\xi, 0}+a_{\xi, 1}\right)>0 .
$$

Let $\alpha$ be a cardinal $<\beta$ such that to each $\xi<\alpha$ we may correlate a cardinal $\beta_{\xi}<\beta$ and have $\sum_{\xi<\alpha}^{*} \beta_{\xi}=\beta$. Then

$$
\text { for any } \eta<\beta \text { there is a } \xi \text { satisfying } \eta \leqq \beta_{\xi}<\beta \text {. }
$$

Let

$$
B_{\xi}=\left\{x \mid x=\prod_{\eta<\beta_{\xi}} a_{\eta, f(\eta)} \text { and } f \in 2^{\beta_{\xi}}\right\} \text { for } \xi<\alpha .
$$

Also for each $\xi<\alpha$, let $\rho_{\xi}=2^{\left(\beta_{\xi}\right)}$, and choose a univalent function $\phi_{\xi}$ on $2^{\beta_{\xi}}$ onto $\rho_{\xi}$. For each $\xi<\alpha$ let $b_{\xi}$ be a function on $\rho_{\xi}$ such that

$$
b_{\xi}\left(\phi_{\xi}(f)\right)=\prod_{\eta<\beta_{\xi}} a_{\eta, f(\eta)}
$$

for each $f \in 2^{\beta_{\xi}}$. We shall write $b_{\xi, \eta}$ for $b_{\xi}(\eta)$ when $\xi<\alpha$ and $\eta<\rho_{\xi}$.

Since $A$ is $(\alpha, \beta)$-distributive,

$$
\prod_{\xi<\alpha} \sum B_{\xi}=\prod_{\xi<\alpha} \sum_{\eta<p_{\xi}} b_{\xi, \eta}=\sum_{o \in G} \prod_{\xi<\alpha} b_{\xi, o(\xi)}
$$

where $G$ is the set of all functions $g$ on $\alpha$ such that $g(\xi)$ is an ordinal $<\rho_{\xi}$. Also by (1), (3) and the distributivity of $A$,

$$
\begin{aligned}
0 & <\prod_{\eta<\beta}\left(a_{\eta, 0}+a_{\eta, 1}\right) \\
& \leqq \prod_{\xi<\alpha} \prod_{\eta<\beta_{\xi}}\left(a_{\eta, 0}+a_{\eta, 1}\right) \\
& =\prod_{\xi<\alpha} \sum_{f \in \beta_{2} \beta_{\xi}} \prod_{\eta<\beta_{\xi}} a_{\eta, f(\eta)} \\
& =\prod_{\xi<\alpha} \sum B_{\xi},
\end{aligned}
$$

so that by (4) there is a $g \in G$ such that 


$$
\prod_{\xi<\alpha} b_{\xi, \theta(\xi)}>0 .
$$

By the definition of $\phi_{\xi}$ we have for each $\xi<\alpha, g(\xi)=\phi_{\xi}(f)$ for some $f \in 2^{\beta_{\xi}}$. Since $g$ is for the moment fixed, this $f$ depends only upon $\xi$, so call it $f_{\xi}$. Now recalling (2), define an $h \in 2^{\beta}$ by the condition that for each $\eta<\beta, h(\eta)=f_{\xi}(\eta)$ where $\xi$ is so chosen that $\beta_{\xi}$ is the least member of $\left\{\beta_{\xi} \mid \xi<\alpha\right\}$ not less than $\eta$. Then by (5) and the definition of the $b_{\xi}$,

$$
\prod_{\eta<\beta} a_{\eta, h(\eta)} \geqq \prod_{\xi<\alpha} b_{\xi, g(\xi)}>0,
$$

which is sufficient for our conclusion.

We noted earlier that if a Boolean algebra is $\alpha$-complete for every $\alpha$ smaller than some singular $\beta$, then that algebra is also $\beta$-complete. We see that Theorem 2.9 is a distributive analogue of a sort. The question arises whether or not the conclusion of Theorem 2.9 still follows if the distributivity assumption upon $A$ is weakened to merely require that $A$ be $(\alpha, \alpha)$-distributive for every $\alpha<\beta$. Theorem 2.11 shows the answer to be negative. For this we shall use the following simple result:

TheOREM 2.10. Suppose that $I$ is a $2^{(\alpha)}$-complete ideal in an $(\alpha, \alpha)$-distributive Boolean algebra $A$. Then $A / I$ is $(\alpha, \alpha)$-distributive.

Proof. By the definitions involved.

Theorem 2.11. Suppose that $\beta$ is a singular, strong limit cardinal. Then there exists a $\beta$-complete Boolean algebra $A$ with the following properties:

(i) For every $\alpha<\beta, A$ is $(\alpha, \alpha)$-distributive.

(ii) For some $\alpha<\beta, A$ is not $(\alpha, \beta)$-distributive.

Proof. Let $B$ be a complete, atomistic Boolean algebra with precisely $2^{(\beta)}$ atoms. Let $I$ be the $\beta$-complete ideal in $B$ generated by the set of all atoms of $B$, and let $A=B / I$. Clearly, (i) follows from Theorem 2.10 , since $B$ is completely distributive. Moreover, one may apply Lemma 2.7 to obtain a sequence $c$ in $B^{\beta \times 2}$ whose image in $A^{\beta \times 2}$ readily shows $A$ not to be $(\beta, \beta)$-distributive. (Alternatively, since $I$ is not $2^{(\beta)}$-complete, $A$ cannot be $(\beta, \beta)$ distributive by Theorem 3.6 (vi) below.) Conclusion (ii) is an immediate consequence of Theorem 2.9.

Several questions naturally arise concerning possible improvement of the above results. For example, in Theorems 2.9 and 2.11 , need $\beta$ be a strong limit number, or is singularity (and infinite) sufficient? Are the hypotheses of completeness necessary in Theorems 2.8 and 2.9? Is it true that any $(\alpha, \beta)$ distributive Boolean algebra is $\left(\alpha, 2^{(\beta)}\right)$-distributive whenever $\beta>\alpha$, even under suitable completeness hypotheses? An affirmative answer to this would not imply that an $(\alpha, \beta)$-distributive Boolean algebra is necessarily $(\alpha, \gamma)$ distributive if $\gamma$ is weakly attainable from $\beta$. To see this, suppose $\beta$ is infinite 
and let $\rho_{0}=\beta, \rho_{1}=2^{\left(\rho_{0}\right)}, \rho_{2}=2^{\left(\rho_{1}\right)}, \rho_{3}=2^{\left(\rho_{2}\right)}, \cdots$, and let $\gamma=\sum_{\xi<\omega}^{*} \rho_{\xi}$. Then $\gamma$ is a singular, strong limit cardinal, weakly attainable from $\beta$, and by Theorem 2.11 there would exist a Boolean algebra which would be $(\sigma, \sigma)$-distributive for every cardinal $\sigma<\gamma$ and yet not $(\alpha, \gamma)$-distributive for some $\alpha<\gamma$.

Having discussed $(\alpha, \beta)$-distributivity when $\beta$ is singular, it would be natural to ask what the situation is when $\beta$ is regular. We know that if $\beta$ is any (infinite) regular cardinal, then there exists a Boolean algebra which is $\alpha$-complete for every $\alpha<\beta$ and yet not $\beta$-complete. Similarly, there exists a Boolean algebra which is $(\alpha, \beta)$-distributive for every $\alpha<\beta$ and yet not $(\beta, \beta)$ distributive $\left.{ }^{8}\right)$.

In $\S 1$ we noted that there is a close tie between atomistic Boolean algebras and fields of sets. It has been known for some time that a very close relationship also exists between the distributivity and atomisticity of a Boolean algebra $\left(^{9}\right)$. Much of our present knowledge of this relationship is codified in the next theorem, for which we shall need two definitions.

DEFinition 2.12. A nonempty collection $R$ of elements of a Boolean algebra $A$ is called a ramification system in $A$ whenever (i) $x$ and $y \in R$ imply that either $x \cdot y=0$ or $x \leqq y$ or $x \geqq y$, and (ii) $x \in R$ implies that $\{y \mid y \in R$ and $x \leqq y\}$ is well ordered with respect to " $\geqq$ ".

Definition 2.13. A field $A$ of sets is $\beta$-complete in the wider sense if the following condition is satisfied: If $X \subseteq A, \kappa(X) \leqq \beta$ and $\sum X$ exists in $A$, then $\sum X=\bigcup X$.

THEOREM 2.14. Let $A$ be a Boolean algebra. The following statements are equivalent:

(i) $A$ is atomistic.

(ii) $A$ is completely distributive.

(iii) For some $\beta, A$ is $(\beta, \beta)$-distributive and $\delta(A)<\beta$.

(iv) For some $\beta, A$ is $(\beta, \beta)$-distributive and the power of every ramification system in $A$ is at most $\beta$,

(v) For some $\beta, A$ is isomorphic to a field of sets which is $\beta$-complete in the wider sense and $\delta(A) \leqq \beta$.

Proof $\left({ }^{10}\right)$. Suppose (i), fix $\alpha$ arbitrarily, let $b$ be a nonzero element of $A$,

(8) This was first proved by E. C. Smith, Jr. and is found in [9]. The proof presented there can be modified to demonstrate the stronger result that for any infinite, regular cardinal $\beta$, there exists a Boolean algebra which is $(\alpha, \gamma)$-distributive for every $\alpha<\beta$ and every $\gamma$ and yet not $(\beta, \beta)$-distributive. A simpler proof of this stronger result has been found by Dana Scott and is given in a note following this paper.

(9) Reference to these ideas is made in [12, pp. 195-197], [1, p. 166], [3], [5] and [14].

(10) The equivalence of conditions (i) and (ii) for complete Boolean algebras was established by Lindenbaum and Tarski and is given by Tarski in [14, Theorem 5]. This result was later extended to arbitrary Boolean algebras by Tarski and was stated in [5, p. 495]. The proof appears here for the first time. The question of the equivalence of (i) and (ii) is mistakenly treated by Enomoto as an open question. A proof that (iv) implies (i) is given in [3, Theorem 3.2]. 
and let $a$ be a member of $A^{\alpha \times \alpha}$ such that $\sum_{\eta<\alpha} a_{\xi, \eta}=b$ for every $\xi<\alpha$. Let $c$ be an atom of $A$ such that $c \leqq b$. Choose an $f \in \alpha^{\alpha}$ such that $c \leqq a_{\xi, f(\xi)}$ for every $\xi<\alpha$. Then $\prod_{\xi<\alpha} a_{\xi, f(\xi)}=0$ is false, which implies that $A$ is $(\alpha, \alpha)$-distributive. Since $\alpha$ is arbitrary, (ii) is established.

Now suppose (iii), and in order to demonstrate that this implies (i), it is sufficient to show that every proper principal ideal in $A$ contains an atom of $A$. Let $0 \neq a \in A$, and suppose that $[a]$ is atomless. For $\xi<\beta$ we shall define ordinals $\alpha_{\xi}$, disjointed subsets $Y_{\xi}$ and $Z_{\xi}$ of $A$, and elements $a_{\xi, \rho}$ of $A$ for $\rho<\alpha_{\xi}$ to satisfy:

(1) $Y_{\xi} \cup Z_{\xi}=\left\{x \mid x=a_{\xi, \rho}\right.$ and $\left.\rho<\alpha_{\xi}\right\}$,

(2) $0 \notin Y_{\xi} \cup Z_{\xi}$,

(3) $\sum\left(Y_{\xi} \cup Z_{\xi}\right)$ exists and equals $a$,

(4) $a_{\xi, \rho} \cdot a_{\xi, \rho^{\prime}}=0$ whenever $\rho^{\prime}<\rho<\alpha_{\xi}$,

(5) $a_{\xi, \rho}<a_{\xi^{\prime}, \rho^{\prime}}$ or $a_{\xi, \rho} \cdot a_{\xi^{\prime}, \rho^{\prime}}=0$ whenever $\xi^{\prime}<\xi, \rho^{\prime}<\alpha_{\xi^{\prime}}, \rho<\alpha_{\xi}$, and $a_{\xi, \rho} \in Y_{\xi}$,

(6) if $g \in \beta^{\xi}, g(\rho)<\alpha_{\rho}$ when $\rho<\xi$, and $\prod_{\rho<\xi} a_{\rho, o(\rho)}=0$ is false, then $a_{\rho, \theta(\rho)} \in Y_{\rho}$ for $\rho<\xi$.

Our definition is by recursion. Let $Y_{0}=\{a\}, a=a_{0,0}, \alpha_{0}=1$, and $Z_{0}=0$; let $\eta$ be $<\beta$ and $>0$, and suppose that we have the $Y_{\xi}, Z_{\xi}$ and $\alpha_{\xi}$ for all $\xi<\eta$, and the $a_{\xi, \rho}$ for $\xi<\eta$ and $\rho<\alpha_{\xi}$. Let

$$
G_{\eta}=\left\{g \mid g \in \beta^{\eta} \text { and } g(\xi)<\alpha_{\xi} \text { when } \xi<\eta\right\} .
$$

For each $g \in G_{\eta}$ such that $\prod_{\xi<\eta} a_{\xi, g(\xi)}=0$ does not hold, let $Y(g)$ be a maximal disjointed set of elements of $A$ satisfying:

(7) $y \in Y(g)$ implies that $0<y<a_{\xi, g(\xi)}$ for all $\xi<\eta$, and

(8) $Y(g)$ has at least two elements.

We can choose $Y(g)$ to satisfy $(8)$; for otherwise, $\prod_{\xi<\eta} a_{\xi, g(\xi)}$ would exist and be an atom $\leqq a$, contrary to supposition. Now let

$$
Y_{\eta}=U\left\{X \mid X=Y(g), g \in G_{\eta} \text {, and } \prod_{\xi<\eta} a_{\xi, g(\xi)}=0 \text { is false }\right\} .
$$

Clearly, for each $g \in G_{\eta}$ such that $\prod_{\xi<\eta} a_{\xi, g(\xi)}=0$ is false, the set $Y(g)$ is disjointed. Furthermore, if $g$ and $g^{\prime}$ are distinct members of $G_{\eta}$ such that $\prod_{\xi<\eta} a_{\xi, 0(\xi)}=0$ and $\prod_{\xi<\eta} a_{\xi, 0^{\prime}(\xi)}=0$ are both false, then $g(\xi) \neq g^{\prime}(\xi)$ for some $\xi<\eta$; and if $b \in Y(g)$ and $c \in Y\left(g^{\prime}\right)$, using the fact that $b \leqq a_{\xi, g(\xi)}$ and $c \leqq a_{\xi, g^{\prime}(\xi)}$, we infer from (4) that $b \cdot c=0$. We therefore see that $Y_{\eta}$ is disjointed.

Now let $Z_{\eta}$ be a maximal disjointed set of nonzero elements of $[a]$ satisfying:

(9) $z \in Z_{\eta}$ implies that $z \cdot y=0$ for every $y \in Y_{\eta}$.

We see that $\sum\left(Y_{\eta} \cup Z_{\eta}\right)$ exists and equals $a$. For otherwise, there would be a nonzero element $b<a$ such that $b \cdot x=0$ for every $x \in Y_{\eta} \cup Z_{\eta}$. But $b \cdot y=0$ for every $y \in Y_{\eta}$ implies that $b \cdot z>0$ for some $z \in Z_{\eta}$, since $Z_{\eta}$ is maximal with respect to (9), a contradiction. We may well order $Y_{\eta} \cup Z_{\eta}$ as $\left\{x \mid x=a_{\eta, \rho}\right.$ and $\left.\rho<\alpha_{\eta}\right\}$, defining $\alpha_{\eta}$. It then follows that we have (1), (2), (3), (4) and (5) for $\eta$. 
Now let $g \in G_{\eta}$ be such that $\prod_{\rho<\eta} a_{\rho, \theta(\rho)}=0$ is false. If $\eta$ is a limit ordinal, then by our inductive hypothesis it follows from (6) that $a_{\rho, \theta(\rho)} \in Y_{\rho}$ for $\rho<\eta$. Suppose that $\eta=\tau+1$ for some ordinal $\tau$. Again by our inductive hypothesis, $a_{\rho, \theta(\rho)} \in Y_{\rho}$ for $\rho<\tau$. Moreover, since $a_{\tau, g(\tau)} \cdot \prod_{\rho<\tau} a_{\rho, \rho(\rho)}=0$ is false and $Y_{\tau} \cup Z_{\tau}$ is disjointed, it follows that $a_{\tau, \theta(\tau)} \in Y_{\tau}$. Consequently, we also have (6) for $\eta$.

The $Y_{\xi}, Z_{\xi}, \alpha_{\xi}$ and $a_{\xi, \rho}$ for $\rho<\alpha_{\xi}$ may therefore be defined as desired for all $\xi<\beta$.

Since each $\sum_{\rho<\alpha \xi} a_{\xi, \rho}$ and $\prod_{\xi<\beta} \sum_{\rho<\alpha \xi} a_{\xi, \rho}$ exist, and this latter product is $a$, and consequently $\neq 0$, the assumption that $A$ is $(\beta, \beta)$-distributive implies that there is a function $f$ on $\beta$ such that $f(\xi)<\alpha_{\xi}$ and $\prod_{\xi<\beta} a_{\xi, f(\xi)} \neq 0$. Consequently, (5) and (6) imply that

$$
a_{\xi, f(\xi)}>a_{\xi+1, f(\xi+1)}>0
$$

for $\xi<\beta$, so that

$$
\left\{x \mid x=a_{\xi, f(\xi)} \cdot\left[a_{\xi+1, f(\xi+1)}\right]^{-} \text {and } \xi<\beta\right\}
$$

is a disjointed set of power $\beta$, contradicting the assumption that $\delta(A)<\beta$. Hence, it follows that $[a]$ is not atomless.

The above construction can be modified to demonstrate that (iv) also implies (i). We shall not explicitly do so. It is obvious that (iii) and (iv) are implied by (ii), so that we see at this point that statements (i) through (iv) are equivalent. Moreover, we may form a field $F$ of sets by letting $x \in F$ if, and only if, for some $a \in A, x=\{b \mid b$ is an atom of $A$ and $b \leqq a\}$. Then (v) easily follows from (i). It remains only to show that (v) implies (i).

Assume (v) and let $\phi$ be an isomorphism of $A$ onto a field $F$ of sets which is $\beta$-complete in the wider sense. Let $S$ be the underlying set of $F$. Let $x \in S$, and let $B$ be a maximal disjointed set of elements $b$ of $A$ satisfying the condition that $b \in B$ only if $x \notin \phi(b)$. Suppose that $\sum B$ does not exist. Then there would be elements $a, c \in A$ such that $a \cdot c=0, a>0, c>0$ and $b \cdot a=b \cdot c=0$ for every $b \in B$. But the maximality of $B$ would imply that $x \in \phi(a)$ and $x \in \phi(c)$, so that $\phi(a): \phi(c)>0$, a contradiction, since $a \cdot c=0$. Consequently, $\sum B$ must exist. Moreover, since $B$ is disjointed, $\kappa(B) \leqq \beta$, so that $\sum \phi(B)=\bigcup_{\phi}(B)$. It then follows that if $x \in \phi(a)$ for some $a \in A$, then $\phi(a)-U_{\phi}(B)$ is an atom of $F$. Therefore, every element of $S$ is contained in an atom of $F$, which is sufficient for our conclusion.

3. Completeness of ideals. This section is primarily a discussion of sufficient conditions for ideals in Boolean algebras to be $\alpha$-complete and closely related results. The majority of the results given here have been known for some time in the case of complete fields of sets.

If $I$ is an ideal in a Boolean algebra $A$ and $I \neq A$, then $I$ can be extended to a prime ideal in $A$; that is, there is a prime ideal $P$ in $A$ such that $I \subseteq P$. We first consider the problem of extending an $\alpha$-complete ideal to an $\alpha$-com- 
plete prime ideal in an $\alpha$-complete Boolean algebra. It is known $\left({ }^{11}\right)$ that every proper principal ideal in a Boolean algebra $A$ can be extended to an $\alpha$-complete prime ideal in $A$ if, and only if, $A$ is isomorphic to an $\alpha$-complete field of sets. A less elementary result is the following:

Theorem 3.1. Suppose that

(i) $A$ is an $\alpha$-complete Boolean algebra,

(ii) $\delta(A-I)<\gamma$ for some cardinal $\gamma$ such that $\alpha \geqq \gamma^{*}$,

(iii) $I$ is an $\alpha$-complete proper ideal in $A$, and

(iv) $A$ is $\left(\boldsymbol{\gamma}, \boldsymbol{\gamma}^{*}\right)$-distributive.

Then there exists an $\alpha$-complete prime ideal $P$ in $A$ such that $I \subseteq P$. Moreover, if $A$ is $\beta$-complete and $I$ is not $\beta$-complete, such a $P$ can be found that it also is not $\beta$-complete.

Proof. The proof follows closely that of [10, Theorem 4.12] and [11, Theorem 3.24]. It should be noted that the essential property of a complete field of sets used in the proofs cited above is distributivity. For completeness we shall give the main ideas.

Suppose that there exists a nonzero element $a$ of $A$ such that $[a] \cap I$ is prime in $[a]$. Then it is easily seen that the ideal $P=\{b \mid b \in A$ and $a \cdot b \in I\}$ is an $\alpha$-complete prime ideal in $A$ which contains $I$. Therefore, suppose that for no $a \in A$ is $[a] \cap I$ prime in $[a]$. Then to each element $a$ of $A-I$ we may correlate two elements $a_{1}$ and $a_{2}$ such that

$$
a=a_{1}+a_{2}, \quad a_{1} \cdot a_{2}=0, \quad a_{1} \notin I, \text { and } a_{2} \notin I .
$$

Now let $h$ be a function defined on $A$ such that $h(a)=a$ if $a \in I$, and $h(a)=a_{1}$ if $a \in A-I$.

For any two ordinals $\eta, \sigma \leqq \gamma$ such that $\eta<\sigma$ and any $f \in 2^{\sigma}$ let $f_{\eta}$ denote the member of $2^{\eta}$ such that $f_{\eta}(\xi)=f(\xi)$ for all $\xi<\eta$. Define a function $X$ on $U_{\xi \leqq \gamma} 2^{\xi}$ by recursion as follows: $X(f)=1$ for $f \in 2^{0}$. Suppose that $\sigma \leqq \gamma, \sigma>0$, and that $X(f)$ is defined for all $f \in U_{\xi \leqq \sigma} 2^{\xi}$. If $\sigma=\eta+1$ for some ordinal $\eta$, for each $f \in 2^{\sigma}$ let $X(f)=h\left(X\left(f_{\eta}\right)\right)$ or $X\left(f_{\eta}\right)-h\left(X\left(f_{\eta}\right)\right)$ according as $f(\eta)=0$ or 1 . If $\sigma$ is a limit number, for each $f \in 2^{\sigma}$ let $X(f)=\prod_{\xi<\sigma} X\left(f_{\xi}\right)$. Thus $X(f)$ may be defined for every $f \in U_{\xi \leqq \gamma} 2^{\xi}$.

By (iv) and an inductive argument one can obtain

$$
\begin{aligned}
1 & =\prod_{\xi<\eta} \sum_{f \in 2^{\xi}} X(f) \\
& =\sum_{f \in 2^{\eta}} \prod_{\xi<\eta} X\left(f_{\xi}\right) \\
& =\sum_{f \in 2^{\eta}} X(f)
\end{aligned}
$$

(11) For $\alpha=\aleph_{0}$, this appears in $[13$, p. 99] and [5, p. 491]. For general $\alpha$, it is given in $[8$, p. 249]. 
for every limit ordinal $\eta \leqq \gamma$. Similarly, if $\eta$ is not a limit number, one easily sees that $1=\sum_{f \in 2} \eta X(f)$.

Now let

$$
M=I \cap\left\{Y \mid Y=X(f) \text { and } f \in \underset{\xi<r}{\bigcup} 2^{\xi}\right\}
$$

Note that

$$
\kappa(M) \leqq \kappa\left\{Y \mid Y=X(f) \text { and } f \in \underset{\xi<\gamma}{\cup} 2^{\xi}\right\} \leqq \sum_{\xi<\gamma}^{*} \kappa(2 \xi) \leqq \gamma^{*} \leqq \alpha .
$$

From (ii) we see that for every $f \in 2^{\gamma}, X\left(f_{\xi}\right) \in M$ for some $\xi<\gamma$. For, otherwise, for some $f \in 2^{\gamma}$ the set

$$
\left\{Y \mid Y=X\left(f_{\xi}\right)-X\left(f_{\xi+1}\right) \text { and } \xi<\gamma\right\}
$$

would be disjointed and of power $\gamma$, contradicting (ii). Then from (1), $\sum M=1$. But from (2), $\sum M \in I$, so that $1 \in I$ and $A=I$, a contradiction.

Consequently, there does exist a nonzero element $a$ of $A$ such that $[a] \cap I$ is prime in $[a]$.

Now suppose that $I$ is not $\beta$-complete. Let

$$
J=\{a \mid a \in A \text { and }[a] \cap I \text { is a } \beta \text {-complete ideal }\} .
$$

We wish to show first that $J$ is an $\alpha$-complete ideal in $A$. Let $\left\{a_{\xi} \mid \xi<\alpha\right\}$ be a subset of $J$. Since $I$ is $\alpha$-complete, it suffices to consider $\left\{a_{\xi} \mid \xi<\alpha\right\} \nsubseteq I$ and to show that $\left[\sum_{\xi<\alpha} a_{\xi}\right] \cap I$ is a $\beta$-complete ideal. Let $B=\left\{b_{\eta} \mid \eta<\beta\right\}$ be a subset of $\left[\sum_{\xi<\alpha} a_{\xi}\right] \cap I$. Then certainly,

$$
\sum B \leqq \sum_{\xi<\alpha} a_{\xi} .
$$

But then also,

$$
\sum B=\sum B \cdot \sum_{\xi<\alpha} a_{\xi}=\sum_{\xi<\alpha} \sum_{\eta<\beta} a_{\xi} \cdot b_{\eta} .
$$

Now since $\left[a_{\xi}\right] \cap I$ is a $\beta$-complete ideal for each $\xi<\alpha$, and each $a_{\xi} \cdot b_{\eta} \in\left[a_{\xi}\right] \cap I$, it follows that $\sum_{\eta<\beta} a_{\xi} \cdot b_{\eta} \in\left[a_{\xi}\right] \cap I \subseteq I$ for each $\xi$. Then by (iii) and (5),

$$
\sum B \in I \text {. }
$$

From (4) and (6) we see that $\sum B \in\left[\sum_{\xi<\alpha} a_{\xi}\right] \cap I$, so that $\sum_{\xi<\alpha} a_{\xi} \in J$. Therefore, $J$ is an $\alpha$-complete ideal in $A$.

Since $I$ is not $\beta$-complete, we note that $1 \notin J$. Now, as in the first part of our argument, we see that there is an element $a$ of $A$ such that $[a] \cap J$ is an $\alpha$-complete prime ideal in $[a]$. Then $a \notin J$. Consequently by (3), $[a] \cap I$ is not a $\beta$-complete ideal, and there is a subset $B$ of $[a] \cap I$ of power at most $\beta$ 
such that $\sum B \notin I$. If $\sum B$ were in $J$, then $\left[\sum B\right] \cap I$ would be a $\beta$-complete ideal which would imply that $\sum B \in I$, a contradiction. Hence, $[a] \cap J$ is $\alpha$-complete but not $\beta$-complete. Set $P=\{b \mid b \in A$ and $a \cdot b \in J\}$, and it easily follows that $P$ is an $\alpha$-complete but not $\beta$-complete prime ideal in $A$ which contains $I$. The theorem is proved.

On careful perusal of the above argument we see that it still holds if we allow $\delta(A-I)=\gamma$ whenever $\delta(A-I)$ is not attained; i.e., the power of no disjointed subset of $A-I$ is $\delta(A-I)$. Furthermore, by virtue of the following theorem, the above restriction on $\delta(A-I)$ could be framed in terms of $\delta(A / I)$.

Theorem 3.2. Let $I$ be an ideal in a Boolean algebra $A$. Then $\delta(A-I)$ $\leqq \delta(A / I)$, and the equality holds if $I$ is $\delta(A-I)$-complete.

Proof. The inequality follows from the definition of the function $\delta$, since disjointed elements of $A-I$ have nonzero, disjointed images in $A / I$.

Now suppose that $\delta(A-I)=\beta$ and that $I$ is $\beta$-complete. Let $\gamma$ be the least cardinal satisfying $\gamma>\beta$, and suppose that there is a subset $\left\{x_{\xi} \mid \xi<\gamma\right\}$ of $A-I$ such that $x_{\xi} \cdot x_{\eta} \in I$ whenever $\xi \neq \eta$ and $\xi, \eta<\gamma$. Such a subset would exist if $\delta(A / I)>\beta$. Define a new subset by letting $y_{0}=x_{0}$ and

$$
y_{\xi}=x_{\xi} \cdot\left[\sum_{\eta<\xi}\left(x_{\xi} \cdot x_{\eta}\right)\right]^{-}
$$

for every $\xi$ such that $0<\xi<\gamma$. Note that the above sums exist since $\xi<\gamma$ implies that $\kappa(\xi) \leqq \beta$ and all the $x_{\xi} \cdot x_{\eta}$ are in $I$. For each $\xi<\gamma$ we have

$$
\begin{aligned}
x_{\xi} & =\sum_{\eta<\xi} x_{\xi} \cdot x_{\eta}+y_{\xi}, \\
\sum_{\eta<\xi} x_{\xi} \cdot x_{\eta} & \in I,
\end{aligned}
$$

and

$$
x_{\xi} \notin I,
$$

whence it follows that $y_{\xi} \notin I$. Then $\left\{y_{\xi} \mid \xi<\gamma\right\}$ is a disjointed subset of $A-I$ with power $>\beta$. This contradicts our assumption that $\delta(A-I)=\beta$.

We remark that the above proof can be modified to show further that $\delta(A-I)$ is attained if, and only if, $\delta(A / I)$ is attained.

The next two theorems are not new; they are specializations of two covering theorems from the general theory of sets given in [17]. When cast in terms of Boolean algebras, their proofs are somewhat simplified. Since our proofs of the principal theorems of this section are dependent upon these theorems, we give them in some detail.

Theorem 3.3. Suppose that

(i) $A$ is a complete, atomistic Boolean algebra,

(ii) the power of the set of all atoms of $A$ is $\beta$, 
(iii) $I$ is an ideal in $A$ such that $\sum I=1$,

(iv) $\delta(A-I) \leqq \alpha$, and

(v) $\beta$ is strongly attainable from $\alpha$.

Then there exists a set $M \subseteq I$ such that $\kappa(M) \leqq \alpha$ and $\sum M=1$.

Proof. Let $K$ be the class of all cardinals $\beta$ for which (i), (ii), (iii) and (iv) always imply the conclusion of this theorem.

Certainly $\alpha \in K$, since in this case we may let $M$ be the set of all atoms of $A$.

Suppose that $\beta \in K$ and $\xi \leqq \beta$, and let $A$ be a Boolean algebra with $\xi$ atoms which satisfies (i), (iii) and (iv). We may imbed $A$ as a principal ideal in a complete, atomistic Boolean algebra $A^{\prime}$ with $\beta$ atoms such that the atoms of $A$ are also atoms of $A^{\prime}$. Let $I^{\prime}=\left\{a \mid a \in A^{\prime}\right.$ and $\left.a \cdot \sum A \in I\right\}$. Since $\beta \in K$, there is a set $M^{\prime} \subseteq I^{\prime}$ such that $\kappa\left(M^{\prime}\right) \leqq \alpha$ and $\sum M^{\prime}=1$. Then the set $\{x \mid x$ $=a \cdot \sum A$ and $\left.a \in M^{\prime}\right\}$ suffices for an $M$ to show that $\xi \in K$.

Consequently, either every cardinal belongs to $K$ (and there is nothing further to demonstrate), or else $\sum_{\beta \in K}^{*} \beta$ exists. For the latter case it is sufficient to show that $\sum_{\beta \in K}^{*} \beta$ is a regular limit number not in $K$, as we shall now do.

Suppose that $\beta \in K$ and that $\gamma$ is the successor to $\beta$. We shall first show that $\gamma \in K$. Let $A$ be a Boolean algebra with $\gamma$ atoms which satisfies (i), (iii) and (iv). Let $B$ be the set of all atoms of $A$. Choose $a \in A^{\beta \times \gamma}$ as follows: Let $\Psi$ be a univalent function on $B$ to $\gamma-\beta$, and for each $\lambda$ such that $\beta \leqq \lambda<\gamma$, let $\phi_{\lambda}$ be a univalent function on $\beta$ to $\lambda$. Let $a_{\xi, \eta}=\sum\left\{b \mid b \in B\right.$ and $\left.\phi_{\Psi(b)}(\xi)=\eta\right\}$ for $\xi<\beta$ and $\eta<\gamma$.

We observe that

$$
a_{\xi, \eta} \cdot a_{\sigma, \eta}=0 \text { whenever } \xi<\sigma<\beta \text { and } \eta<\gamma ;
$$

and

$$
a_{\xi, \eta} \cdot a_{\xi, \sigma}=0 \text { whenever } \xi<\beta \text { and } \eta<\sigma<\gamma .
$$

We now show that

$$
\text { there exists a } \rho<\gamma \text { such that } a_{\xi, \rho} \in I \text { for every } \xi<\beta \text {. }
$$

If this were not the case, for each $\eta<\gamma$ there would be a $\xi(\eta)<\beta$ such that $a_{\xi(\eta), \eta} \notin I$. But since $\gamma$ is regular, there would then be a set $T \subseteq \gamma$ and a $\xi<\beta$ such that $\xi(\eta)=\xi$ for every $\eta \in T$ and $\kappa(T)=\gamma$. Then $\left\{x \mid x=a_{\xi(\eta), \eta}\right.$ and $\left.\eta \in T\right\}$ would be disjointed by (2), and we arrive at a contradiction by (iv).

Now consider any $b \in B$ such that $\rho<\Psi(b)$. By the definition of the $\phi_{\lambda}$ there is a $\xi<\beta$ such that $\phi_{\Psi(b)}(\xi)=\rho$. Consequently, $b \leqq a_{\xi, \rho}$, so that $\kappa\{b \mid b \in B$ and $\left.b \cdot \sum_{\xi<\beta} a_{\xi, \rho}=0\right\} \leqq \kappa(\rho)=\beta$.

Let $A^{\prime}$ be the complete, atomistic subalgebra of $A$ with $\left\{a \mid a=a_{\xi, \rho}\right.$ and $\xi<\beta\} \cup\left\{b \mid b \in B\right.$ and $\left.b \cdot \sum_{\xi<\beta} a_{\xi, \rho}=0\right\}$ as atoms. Then the power of the set of atoms of $A^{\prime}$ is $\leqq \beta+\kappa(\rho) \leqq \beta$. On setting $I^{\prime}=I \cap A^{\prime}$, we see that since $\beta \in K$, 
there exists a set $M \subseteq I^{\prime} \subseteq I$ such that $\kappa(M) \leqq \alpha$ and $\sum M=1$. Consequently, $\gamma \in K$; that is, the successor to any member of $K$ is also a member of $K$. This implies that $\sum_{\beta \in K}^{*} \beta$ is a limit number and does not belong to $K$.

Now suppose that $\gamma$ is a cardinal such that $\gamma<\sum_{\beta \in K}^{*} \beta$, and to each $\xi<\gamma$ is correlated a $\rho_{\xi}$ such that $\rho_{\xi}<\sum_{\beta \in K}^{*} \beta$. Note that $\gamma \in K$ and $\rho_{\xi} \in K$ for $\xi<\gamma$. Let $A$ be a Boolean algebra with $\sum_{\xi<\gamma}^{*} \rho_{\xi}$ atoms which satisfies (i), (iii) and (iv). Let the set of atoms of $A$ be partitioned into sets $B_{\xi} \neq 0$ for $\xi<\gamma$ such that $\kappa\left(B_{\xi}\right) \leqq \rho_{\xi}$ and $B_{\xi} \cap B_{\eta}=0$ for $\xi<\eta<\gamma$. Let $U=\left\{\xi \mid \xi<\gamma\right.$ and $\left.\sum B_{\xi} \in I\right\}$ and $V=\left\{\xi \mid \xi<\gamma\right.$ and $\left.\sum B_{\xi} \notin I\right\}$. We observe that $\sum B_{\xi} \cdot \sum B_{\eta}=0$ whenever $\xi<\eta<\gamma$, so that by (iv), $\kappa(V) \leqq \alpha$.

For each $\xi \in V$ let $A_{\xi}$ be the principal ideal in $A$ generated by $B_{\xi}$. Each such $A_{\xi}$ may be considered as a complete, atomistic Boolean algebra with $B_{\xi}$ as its set of atoms. Let $I_{\xi}=A_{\xi} \cap I$ for $\xi \in V$. Since we assume that $\kappa\left(B_{\xi}\right) \in K$, we can obtain sets $M_{\xi} \subseteq I_{\xi}$ such that $\kappa\left(M_{\xi}\right) \leqq \alpha$ and $\sum M_{\xi}=\sum A_{\xi}$ for $\xi \in V$. Let $A^{\prime}$ be the complete, atomistic Boolean algebra formed by

$$
\left\{\sum X \mid X \subseteq\left\{\sum A_{\xi} \mid \xi \in U\right\}\right\}
$$

with the operations of + and - in $A^{\prime}$ just those of $A$ restricted to this set of elements. Note that $\left\{X \mid X=\sum A_{\xi}\right.$ and $\left.\xi \in U\right\}$ is the set of atoms of $A^{\prime}$. Since $\gamma \in K, \kappa(U) \in K$, so that we can find a set $M^{\prime} \subseteq I \cap A^{\prime}$ such that $\kappa\left(M^{\prime}\right) \leqq \alpha$ and $\sum M^{\prime}=\sum_{\xi \in U}\left(\sum A_{\xi}\right)$.

Let $M=M^{\prime} \cup \bigcup_{\xi \in V} M_{\xi}$. Then $M \subseteq I$ and $\kappa(M)=\kappa\left(M^{\prime}\right)+\sum_{\xi \in V}^{*} \kappa\left(M_{\xi}\right) \leqq \alpha$ and $\sum M=1$, so that $\sum_{\xi<\gamma}^{*} \rho_{\xi} \in K$. This implies that $\sum_{\xi<\gamma}^{*} \rho_{\xi}<\sum_{\beta \in K}^{*} \beta$ and, consequently, $\sum_{\beta \in K}^{*} \beta$ must be regular.

THEOREM 3.4. Suppose that

(i) $A$ is a complete, atomistic Boolean algebra,

(ii) the power of the set of all atoms of $A$ is $\beta$,

(iii) $I$ is an ideal in $A$ such that $\sum I=1$,

(iv) $\delta(A-I)<\gamma$ for some cardinal $\gamma$ such that $\alpha \geqq \gamma^{*}$, and

(v) $\beta$ is weakly attainable from $\alpha$.

Then there exists a set $M \subseteq I$ such that $\kappa(M) \leqq \alpha$ and $\sum M=1$.

Proof. Let $K$ be the class of all cardinals $\beta$ for which (i), (ii), (iii) and (iv) always imply the conclusion of this theorem. As in Theorem 3.3, $\alpha \in K$ and either every cardinal belongs to $K$, or else $\sum_{\beta \in K}^{*} \beta$ is a regular limit number which does not belong to $K$, and $\xi \in K$ whenever $\xi<\beta$ and $\beta \in K$. It suffices to show that if $\eta \in K$, then $2^{(\eta)} \in K$.

Let $\rho=2^{(\eta)}$, and suppose that there exists an ideal $I$ in $A$ which satisfies (iii) and (iv) and yet for every $M \subseteq I$ with $\kappa(M) \leqq \alpha, \sum M<1$. Then there exists a proper $\alpha$-complete ideal $J$ in $A$ which contains $I$. We may apply Theorem 3.1 (with $\beta=\rho$ ) to obtain an $\alpha$-complete prime ideal $P$ which contains $J$ (and $I$ ) and is not $\rho$-complete. If $\rho$ is not weakly attainable from $\alpha$, 
there is nothing more to prove. In case $\rho$ is weakly attainable from $\alpha$, by $[10$, Theorem 3.9], or (alternatively by Corollary 3.7 of this paper) the ideal $P$ is $\rho$-complete, a contradiction. Consequently, it must be that $2^{(\eta)}=\rho \in K$, and the theorem is completely proved.

Theorem 3.5. Suppose that $I$ is an ideal in a Boolean algebra $A$ such that for every disjointed subset $Y$ of $I$ with $\kappa(Y) \leqq \beta, \sum Y$ exists in $A$. Then for every subset $X$ of $I$ with $\kappa(X) \leqq \beta, \sum X$ exists in $A$.

Proof. Our proof is by induction on the set of all cardinal numbers $\leqq \beta$. Certainly every finite sum of elements of $I$ exists. Suppose that for some $\alpha \leqq \beta$ we know that for every $X \subseteq I$ such that $\kappa(X)<\alpha, \sum X$ exists.

Let $X^{\prime}=\left\{x_{\xi} \mid \xi<\alpha\right\} \subseteq I$. Define the set $Y=\left\{y_{\xi} \mid \xi<\alpha\right\}$ by letting $y_{0}=x_{0}$ and $y_{\xi}=x_{\xi} \cdot\left[\sum_{\eta<\xi} x_{\eta}\right]^{-}$for $0<\xi<\alpha$. Then $Y$ is disjointed. Moreover, for each $\xi<\alpha, y_{\xi} \leqq x_{\xi} \in I$, so that $y_{\xi} \in I$ and $\sum Y$ exists in $A$.

Suppose that for some $\rho$ such that $0<\rho<\alpha$, we know that $x_{\Downarrow} \leqq \sum Y$ for every $\eta<\rho$. Then

$$
x_{\rho} \cdot \sum_{\eta<p} x_{\eta} \leqq \sum Y
$$

and

$$
x_{\rho} \cdot\left[\sum_{\eta<\rho} x_{\eta}\right]^{-}=y_{\rho} \leqq \sum Y
$$

so that

$$
x_{\rho}=x_{\rho} \cdot \sum_{\eta<\rho} x_{\eta}+x_{\rho} \cdot\left[\sum_{\eta<\rho} x_{\eta}\right]^{-} \leqq \sum Y
$$

Therefore, it follows that $x_{\xi} \leqq \sum Y$ for all $\xi<\alpha$. Now since $y_{\xi} \leqq x_{\xi}$ for $\xi<\alpha$, we see that $\sum X^{\prime}$ exists and, in fact, is $\sum Y$.

We have shown that the sum of every subset of $I$ with power $\alpha$ exists in $A$. Our conclusion follows by induction.

Theorem 3.6(12). Suppose that

(i) $A$ is a $\beta$-complete Boolean algebra,

(ii) $I$ is an $\alpha$-complete ideal in $A$, and

(iii) $\beta$ is weakly attainable from $\alpha$.

Suppose further that one of the following three conditions is satisfied:

(iv) $2^{(\delta(\mathbf{A}-I))} \leqq \alpha$.

(v) $A / I$ is isomorphic to an $\alpha$-complete field of sets.

(vi) $A / I$ is $(\gamma, \gamma)$-distributive for some cardinal $\gamma$ satisfying $2^{(\gamma)} \geqq \beta$.

Then $I$ is $\beta$-complete.

(12) The portion of this theorem assuming (vi) has also been found by Pierce. See [6]. 
Proof. The conclusion is obvious if $\beta \leqq \alpha$, so assume that $\alpha<\beta$.

Assume first that (iv) holds, and let $B$ be a disjointed subset of $I$ such that $0 \notin B$ and $\kappa(B) \leqq \beta$. From Theorem 3.5 we see that it is sufficient to show that $\sum B \in I$. Let $A^{\prime}$ be the complete, atomistic Boolean algebra formed by $\left\{Y \mid Y=\sum X\right.$ and $\left.X \subseteq B\right\}$, where the operations in $A^{\prime}$ are those of $A$ restricted to this set; and let $I^{\prime}=I \cap A^{\prime}$. We may apply Theorem 3.4 to $A^{\prime}$ and $I^{\prime}$ and thereby obtain a set $M \subseteq I$ such that $\kappa(M) \leqq \alpha$ and $\sum M=\sum B$. Since $I$ is $\alpha$-complete, we have $\sum B \in I$ as desired.

Now assume that (v) holds. There exists an isomorphism $\phi$ of $A / I$ onto an $\alpha$-complete field of subsets of some set $S$. Again let $B$ be an arbitrary subset of $I$ of power at most $\beta$, and we shall again show that $\sum B \in I$. Certainly $0=\phi\left(\sum(B / I)\right) \leqq \phi\left(\left(\sum B\right) / I\right)$, so suppose that there is an element $x$ of $S$ such that $x \in \phi\left(\left(\sum B\right) / I\right)-\phi\left(\sum(B / I)\right)$. Define $P=\{a \mid a \in A$ and $x \notin \phi(a / I)\}$. Suppose that $C$ is any subset of $P$ of power at most $\alpha$. Then by (ii),

$$
\phi\left(\left(\sum C\right) / I\right)=\phi\left(\sum(C / I)\right)=\bigcup_{c \in C} \phi(c / I),
$$

since we assume that $\phi$ is an isomorphism of $A / I$ onto an $\alpha$-complete field of sets. Consequently, $\sum C \in P$, and it follows that $P$ is an $\alpha$-complete ideal in $A$. Moreover, $P$ is prime; for if $a \notin P$, then $x \in \phi(a / I)$, so that $x \notin \phi(\bar{a} / I)$, which implies that $\bar{a} \in P$. Then by the portion of this theorem already proved (for $I=P$ ) under the assumption (iv), $P$ is $\beta$-complete. But then $B \subseteq I \subseteq P$ and $\kappa(B) \leqq \beta$ imply that $\sum B \in P$, or, equivalently, that $x \in \phi\left(\left(\sum B\right) / I\right)$, a contradiction of our original supposition on $x$.

Now assume that (vi) is true. Let us assume that $\mu<\nu \leqq 2^{(\mu)}, \nu \leqq \beta$, $\alpha \leqq \mu \leqq \gamma$, and that $I$ is $\mu$-complete. Upon examination of the definition of weak attainability, we see that it suffices to show that $I$ is $\nu$-complete. Let $B$ be a disjointed subset of $I$ of power at most $\nu$; we shall show that $\sum B \in I$. Let $\phi$ be a univalent function on $B$ into $\{X \mid X \subseteq \mu\}$, and let $a$ be the member of $A^{\mu \times 2}$ such that for $\xi<\mu$,

$$
\begin{aligned}
& a_{\xi, 0}=\sum\{b \mid b \in B \text { and } \xi \in \phi(b)\}, \\
& a_{\xi, 1}=\sum\{b \mid b \in B \text { and } \xi \in \phi(b)\} .
\end{aligned}
$$

Clearly, $\sum B=a_{\xi, 0}+a_{\xi, 1}$ for each $\xi<\mu$. Since we assume $I$ to be $\mu$-complete, and $\mu \leqq \gamma$, we have by (vi)

$$
\begin{aligned}
\left(\sum B\right) / I & =\left[\prod_{\xi<\mu}\left(a_{\xi, 0}+a_{\xi, 1}\right)\right] / I=\prod_{\xi<\mu}\left[\left(a_{\xi, 0}\right) / I+\left(a_{\xi, 1}\right) / I\right] \\
& =\sum_{f \in 2^{\mu}} \prod_{\xi<\mu}\left(a_{\xi, f(\xi)}\right) / I=\sum_{f \in 2^{\mu}}\left(\prod_{\xi<\mu} a_{\xi, f(\xi)}\right) / I .
\end{aligned}
$$

Now let $f$ be a fixed but arbitrary member of $2^{\mu}$, and consider the set 
$Y=\{\xi \mid \xi<\mu$ and $f(\xi)=0\}$. Suppose that for some $b \in B, \phi(b)=Y$. By an argument quite analogous to that used to establish Lemma 2.7, we see that $\prod_{\xi<\mu} a_{\xi, f(\xi)}=b$. Now suppose that for every $b \in B, \phi(b) \neq Y$. Then for any $b \in B$ there is an $\eta<\mu$ such that either $\eta \in Y-\phi(b)$ or $\eta \in \phi(b)-Y$, so that $b \cdot a_{\eta, f(\eta)}=0$. Consequently, $\prod_{\xi<\mu} a_{\xi, f(\xi)}=0$. Therefore, we see that in any case,

$$
\prod_{\xi<\mu} a_{\xi, f(\xi)} \in I .
$$

From (1) and (2) we conclude that

$$
\left(\sum B\right) / I=\sum_{f \in 2^{\mu}}\left(\prod_{\xi<\mu} a_{\xi, f(\xi)}\right) / I=0,
$$

which implies that $\sum B \in I$. It follows that $I$ is $\nu$-complete by Theorem 3.5.

In the above proof under the assumption (v) we noted the application of this theorem to prime ideals. We now formally state this as a corollary.

Corollary 3.7. Suppose that (i), (ii) and (iii) of Theorem 3.6 are satisfied and that $I$ is a prime ideal in $A$. Then $I$ is $\beta$-complete.

Observe that this also follows from Theorem 3.6 (vi), and consequently is independent of Theorem 3.4. A weak form of this corollary is given by Sikorski in [8, p. 254]. For fields of sets, see also [10, Theorem 3.9] and [11, Theorem 3.19]. The following theorem has its counterpart for fields of sets in [11, Theorems 3.23 and 3.25].

Theorem 3.8. Suppose that $I$ is an ideal in a $\beta$-complete Boolean algebra $A$ such that $\delta(A-I) \leqq \alpha$. Suppose further that one of the two following conditions is satisfied:

(i) $\beta$ is weakly attainable from $\alpha$ and $I$ is $2^{(\alpha)}$-complete.

(ii) $\beta$ is strongly attainable from $\alpha$ and $I$ is $\alpha$-complete.

Then $I$ is $\beta$-complete.

Proof. In case (i) is true, the conclusion follows from Theorem 3.6. Assume that (ii) holds, and let $B$ be a disjointed subset of $I$ of power at most $\beta$. Using Theorem 3.3 and an argument analogous to that used to prove Theorem 3.6 under its assumption (iv), we conclude that $\sum B \in I$, whence $I$ is $\beta$-complete by Theorem 3.5.

Observe that if $A$ is complete and $\delta(I)=\beta$, then this theorem implies that $I$ is also complete (and principal). Moreover, the proof given for Theorem 3.6 under its assumption (iv) holds verbatim if, instead of requiring $A$ to be $\beta$-complete, we require the weaker condition that every disjointed subset of $I$ with power at most $\beta$ has a sum in $A$. The hypothesis of Theorem 3.8 may be weakened similarly. It remains an open question whether or not "strongly attainable" can be replaced by "weakly attainable" in hypothesis (ii) of the above theorem and in hypothesis (v) of Theorem 3.3. In fact, just how essen- 
tial any concept of attainability is to the results of this section is not known. We can say the following:

ThEOREM 3.9. If there exists an atomless, complete Boolean algebra $A$ with an $\alpha$-complete prime ideal $P$, then there exists an inaccessible number $\beta$ such that $\alpha<\beta \leqq \delta(A)$.

Proof. Suppose the contrary. Then the sum of every disjointed set of elements of $P$ is also in $P$. Theorem 3.5 then implies that $P$ is complete, so that $A$ has an atom. The result immediately follows, since $A$ is assumed to be atomless.

The collection of all regular open sets of any Hausdorff space without isolated points forms a complete, atomless Boolean algebra. The above theorem indicates the difficulty of determining whether such an algebra has a countably complete prime ideal. It may be, of course, that such Boolean algebras do not contain countably complete prime ideals regardless of the existence or nonexistence of inaccessible numbers. The general answer to this is not known.

Since a Boolean algebra $A$ is isomorphic to a $\beta$-complete field of sets only if every ideal in $A$ is contained in a $\beta$-complete prime ideal, any statement concerning such an isomorphism is really a statement about completeness of ideals. We observed an example of this relationship in Theorem 3.6, and now illustrate it further.

Theorem 3.10. Suppose that $\beta$ is weakly attainable from $\alpha$. Then every $\alpha$-complete field of sets which is $\beta$-complete as a Boolean algebra is also a $\beta$-complete field of sets.

Proof. Let $S$ be the underlying set of an $\alpha$-complete field of sets which, as a Boolean algebra $A$, is $\beta$-complete. Let $B$ be an arbitrary subset of $A$ of power at most $\beta$. Suppose that there exists an $x \in S$ such that $x \in \sum B-\cup B$. Define $P=\{a \mid a \in A$ and $x \notin a\}$, and, as in the proof of Theorem 3.6, note that $P$ is an $\alpha$-complete prime ideal in $A$. Consequently, $P$ is $\beta$-complete, so that $B \subseteq P$ implies that $x \notin \sum B$, a contradiction. Hence, $\sum B=\bigcup B$, and it follows that the given field of sets is $\beta$-complete.

In the light of the above theorem, note the following example: Let $S$ be a countable set and $A$ the Boolean algebra of all subsets of $S$. Let $P$ be a nonprincipal prime ideal in $A$, and let $x$ be some point not in $S$. Then

$$
P \cup\{y \mid y=a \cup\{x\} \text { and } a \in A-P\}
$$

forms a field of sets which is not complete, and yet as a Boolean algebra is both complete and atomistic.

We saw in Theorem 2.14 that distributivity is closely tied with atomisticity and isomorphs of fields of sets. It is further known that $(\alpha, \alpha)$-distributivity is sufficient for an $\alpha$-complete Boolean algebra to be an $\alpha$-homomorphic 
image of an $\alpha$-complete field of $\operatorname{sets}\left({ }^{13}\right)$. We can now show that $(\alpha, \alpha)$-distributivity is not sufficient to imply that such a Boolean algebra is isomorphic to an $\alpha$-complete field of $\operatorname{sets}\left({ }^{14}\right)$.

THEOREM 3.11. Suppose that a cardinal $\gamma$ is weakly attainable from $\alpha$ and that $\gamma \geqq \alpha$. Then there is a $2^{(\gamma)}$-complete, $(\gamma, \gamma)$-distributive Boolean algebra which is not isomorphic to any $\alpha$-complete field of sets.

Proof. Let $\rho=2^{(\gamma)}, \beta=2^{(\rho)}$, and let $S$ be any set of power $\beta$. Let $A$ be the Boolean algebra of all subsets of $S$, and $I$ the $2^{(\gamma)}$-complete ideal of all subsets of power at most $2^{(\gamma)}$. Then $I$ is not $\beta$-complete and, therefore, $A / I$ cannot be isomorphic to any $\alpha$-complete field of sets by Theorem 3.6. Note that $A / I$ is $(\gamma, \gamma)$-distributive by Theorem 2.10 .

4. Completeness of factor algebras and applications to measure. We have of ten used the fact that if $I$ is a $\beta$-complete ideal in a $\beta$-complete Boolean algebra $A$, then $A / I$ is $\beta$-complete. By Theorem 3.5 it follows that if $\delta(A / I)$ $\leqq \beta$, or equivalently, since $I$ is $\beta$-complete, $\delta(A-I) \leqq \beta$, then $A / I$ is complete. We shall show that the same conclusion can be obtained under slightly weaker conditions upon $I$, and indicate some implications of this.

Lemma 4.1. Suppose that

(i) $A$ is a $\beta$-complete Boolean algebra,

(ii) $I$ is an ideal in $A$ which is $\alpha$-complete for every $\alpha<\beta$, and

(iii) $X$ is a disjointed subset of $A / I$ such that $\kappa(X) \leqq \beta$ and $\sum X$ exists.

Then $\sum Y$ exists for every $Y \subseteq X$.

Proof. If $\kappa(Y)=\alpha$ for some $\alpha<\beta, \sum Y$ certainly exists, for in this case (i) and (ii) imply that $A / I$ is $\alpha$-complete. Therefore, assume that $\kappa(Y)=\beta$, and well order $X$ as $\left\{x_{\xi} \mid \xi<\beta\right\}$ where $x_{\xi} \neq x_{\eta}$ when $\xi<\eta<\beta$. Also, let $B=\left\{b_{\xi} \mid \xi<\beta\right\}$ be any set of antecedents for $X$ in $A$ such that $b_{\xi} / I=x_{\xi}$ for $\xi<\beta$. Set $a_{0}=b_{0}$, and for $0<\xi<\beta$ set

$$
a_{\xi}=b_{\xi} \cdot\left[\sum_{\eta<\xi} b_{\eta}\right]^{-}
$$

and note that

$$
b_{\xi} \leqq \sum_{\eta \leqq \xi} a_{\eta}
$$

and $a_{\xi} \cdot a_{\eta}=0$ when $\xi<\eta<\beta$. By virtue of (iii), $b_{\xi} \cdot b_{\eta} \in I$ whenever $\xi<\eta<\beta$, so that

(13) E. C. Smith, Jr. has shown that a distributive condition weaker than $(\alpha, \alpha)$-distributivity is sufficient for an $\alpha$-complete Boolean algebra to be an $\alpha$-homomorphic image of an $\alpha$-complete field of sets. More recently, C. C. Chang has given a necessary and sufficient condition for such a homomorphism. See [2], [9] and [6].

(14) For $\alpha$ strongly attainable from $\aleph_{0}$, this is stated in [4]. For the case $\alpha=\aleph_{0}$, see [13, p. 99] and $[5$, p. 492]. See also [6]. 


$$
b_{\xi} \cdot a_{\eta} \in I \text { when } \xi \neq \eta \text { and } \xi, \eta<\beta \text {. }
$$

Setting

$$
\begin{aligned}
& y=\sum\left\{a_{\xi} \mid \xi<\beta \text { and } x_{\xi} \in Y\right\}, \\
& z=\sum\left\{a_{\xi} \mid \xi<\beta \text { and } x_{\xi} \in Y\right\}
\end{aligned}
$$

we have

$$
y \cdot z=0 .
$$

Now by (1), (2) and (3), for each $\xi<\beta$ such that $x_{\xi} \in Y$,

$$
\begin{aligned}
\bar{y} \cdot b_{\xi} & =\bar{y} \cdot b_{\xi} \cdot \sum_{\eta \leqq \xi} a_{\eta} \\
& =\bar{y} \cdot b_{\xi} \cdot\left(\sum\left\{a_{\eta} \mid \eta \leqq \xi \text { and } x_{\eta} \in Y\right\}+\sum\left\{a_{\eta} \mid \eta<\xi \text { and } x_{\eta} \notin Y\right\}\right) \\
& =\bar{y} \cdot b_{\xi} \cdot \sum\left\{a_{\eta} \mid \eta<\xi \text { and } x_{\eta} \notin Y\right\} \\
& =\sum\left\{c \mid c=b_{\xi} \cdot a_{\eta}, \eta<\xi \text { and } x_{\eta} \in Y\right\} \in I,
\end{aligned}
$$

since $\kappa(\xi)<\beta$ and $I$ is $\alpha$-complete for every $\alpha<\beta$. Consequently, if $\xi<\beta$ is such that $x_{\xi} \in Y$, then $x_{\xi}=b_{\xi} / I \leqq y / I$. In an analogous manner it can be shown that $x_{\xi}=b_{\xi} / I \leqq z / I$ when $x_{\xi} \notin Y$. Then $\sum X \leqq y / I+z / I$. But by (4), $(y / I) \cdot(z / I)=0$; hence it follows that $(y / I) \cdot \sum X=\sum Y$ and $(z / I) \cdot \sum X$ $=\sum(X-Y)$.

TheOREM 4.2. Suppose that

(i) $A$ is a $\beta$-complete Boolean algebra,

(ii) $I$ is an ideal in $A$ which is $\alpha$-complete for every $\alpha<\beta$, and

(iii) $\delta(A / I) \leqq \beta$.

Then $A / I$ is complete.

Proof. Let $Y$ be an arbitrary disjointed subset of $A / I$. It is sufficient to show that $\sum Y$ exists. Let $X$ be any maximal disjointed subset of $A / I$ which contains $Y$. The maximality of $X$ insures that $\sum X$ exists and is 1 . By (iii), $\kappa(X) \leqq \beta$, so that $\sum Y$ exists by the preceding lemma.

Corollary 4.3. If $I$ is an ideal in a countably complete Boolean algebra $A$ such that $\delta(A / I) \leqq \boldsymbol{N}_{0}$, then $A / I$ is complete.

Proof. Obvious.

Theorem 4.4. Suppose that I is an ideal in a countably complete Boolean algebra $A$. Then $\kappa(A / I) \neq \boldsymbol{\aleph}_{0}$.

Proof. Suppose the contrary, and let $X$ be any disjointed subset of $A / I$ such that $\kappa(X)=\boldsymbol{\aleph}_{0}$. Certainly such an $X$ exists, since our supposition implies that $A / I$ is infinite. By Corollary $4.3, A / I$ is complete. Then $\left\{z \mid z=\sum Y\right.$ and $Y \subseteq X\}$ is a set of $2^{\left(\boldsymbol{N}_{0}\right)}$ distinct elements of $A / I$, contradicting the supposition. 
This theorem implies that no Boolean algebra with power $\boldsymbol{\aleph}_{0}$ is a homomorphic image of a complete, atomistic Boolean algebra $\left({ }^{15}\right)$.

Definition 4.5. A real valued function $\mathfrak{m}$ defined on a Boolean algebra $A$ is called a (finitely additive) measure on $A$ if it satisfies the following conditions:

(i) $0 \leqq \mathfrak{m}(a)$ for all $a \in A$,

(ii) $\mathfrak{m}(1)=1$, and

(iii) $\mathfrak{m}(a+b)=\mathfrak{m}(a)+\mathfrak{m}(b)$ whenever $a, b \in A$ and $a \cdot b=0$.

$A$ measure $\mathfrak{m}$ is called countably complete if (iii) is replaced by (iii') $\mathfrak{m}\left(\sum_{\xi<\aleph_{0}} a_{\xi}\right)$ $=\sum_{\xi<\aleph_{0}} \mathfrak{m}\left(a_{\xi}\right)$ for eiery disjointed, countable sequence $a \in A^{\aleph_{0}}$ such that $\sum_{\xi<\aleph_{0}} a_{\xi}$ exists. A measure function is called two-valued if its range is 2.

Lemma 4.6. Suppose that $\mathfrak{m}$ is a measure on a Boolean algebra $A$, and that $I$ is the ideal of all elements $a \in A$ such that $\mathfrak{m}(a)=0$. Then $\delta(A / I) \leqq \aleph_{0}$.

Proof. This has been proved by A. Tarski for fields of sets in [10, Theorem 5.2 ]. Since a Boolean algebra is isomorphic to a field of sets, our general result follows.

Theorem 4.7. Suppose that $\mathfrak{m}$ is a measure on a countably complete Boolean algebra $A$, and that $I$ is the ideal of all elements $a \in A$ such that $\mathfrak{m}(a)=0$. Then $A / I$ is complete.

Proof. By Lemma 4.6, $\delta(A / I) \leqq \boldsymbol{\aleph}_{0}$. Therefore, apply Corollary 4.3.

Theorem 3.5 implies the well known fact that the Boolean algebra of all Lebesgue measurable subsets of the real line modulo those subsets of measure zero is complete (since the ideal of sets of measure zero is countably complete). However, Theorem 4.7 is actually stronger than Theorem 3.5 applied in this way, since there exist measures on Boolean algebras such that the ideal of elements of measure zero is not countably complete.

It is known $\left({ }^{16}\right)$ that if $A$ is the Boolean algebra of all subsets of an infinite set $S$ whose power is strongly attainable from $\boldsymbol{\aleph}_{0}$, and $I$ is a proper, countably complete ideal in $A$ which contains all of the one-point subsets of $S$, then there is no countably additive measure on $A / I$. This can be obtained from Theorem 3.8 on setting $\alpha=\boldsymbol{\aleph}_{0}$ and considering the ideal of all elements of measure zero for a contradiction. Moreover, if the power of $S$ is weakly attainable from $\boldsymbol{\aleph}_{0}$, then it is known $\left({ }^{17}\right)$ that there is no countably additive, twovalued measure on $A / I$. This can be obtained from Corollary 3.7, since for any two-valued measure on a Boolean algebra the ideal of all elements of measure zero is prime.

We extend these results to more general Boolean algebras.

(15) This is a solution of a problem proposed by R. Dilworth.

(16) See [18].

(17) This is a consequence of [10, Theorem 3.9]. These ideas are also discussed in [5]. 
TheOREM 4.8. Suppose that I is a countably complete, proper ideal in a complete Boolean algebra $A$ such that $\delta(A)$ is strongly attainable from $\boldsymbol{\aleph}_{0}$. Suppose further that one of the two following conditions is satisfied:

(i) $\sum I=1$.

(ii) $\delta([a])>\aleph_{0}$ for every nonzero $a \in A$.

Then there is no countably additive measure on $A / I$.

Proof. Suppose that there is a countably additive measure on $A / I$. Then the antecedent in $A$ of the ideal in $A / I$ of all elements of measure zero is a countably complete ideal $J$. Moreover, $I \subseteq J$ and $J \neq A$. Since $\delta(A / J) \leqq \boldsymbol{\aleph}_{0}$ by Lemma 4.6, we may apply Theorem 3.8 to $A$ and $J$ with $\alpha=\boldsymbol{\aleph}_{0}$. Then $J$ is principal. If (i) is assumed, we have $J=A$, a contradiction. If we assume (ii), $J \neq A$ implies that there is an element $a \in A$ such that $a>0$ and $[a] \cap J=\{0\}$. Then (ii) implies that $\delta(A / J)>\boldsymbol{\aleph}_{0}$, which is not true.

We remark that this theorem implies that there is no countably additive measure $\mathrm{m}$ on a complete Boolean algebra $A$ such that $\delta(A)$ is strongly attainable from $\boldsymbol{\aleph}_{0}$ and $\sum\{a \mid a \in A$ and $\mathfrak{m}(a)=0\}=1$.

TheOREM 4.9. Suppose that

(i) $A$ is a complete Boolean algebra,

(ii) I is a countably complete, proper ideal in $A$,

(iii) $\delta(A)$ is weakly attainable from $\boldsymbol{\aleph}_{0}$, and

(iv) $\sum I=1$.

Then there is no countably additive, two-valued measure on $A / I$.

Proof. If there were a countably additive, two-valued measure on $A / I$, then the antecedant in $A$ of the ideal of all elements of $A / I$ of measure zero would be a countably complete prime ideal $J$. It would then follow from Corollary 3.7 and (iii) that $J$ was principal and, therefore, identical with $A$ by (iv). This is a contradiction.

If $A$ is a Boolean algebra that satisfies (i) and (iii) of the above theorem, then there is no countably additive, two-valued measure on $A$ such that the measure of every atom is zero. Thus, if $A$ is atomless, there is no countably additive, two-valued measure on $A$ at all.

\section{BIBLIOGRAPHY}

1. G. Birkhoff, Lattice theory, Amer. Math. Soc. Colloquium Publications, rev. ed., vol. 25, 1948.

2. C. C. Chang, Bull. Amer. Math. Soc. Abstract 61-4-579 (1955).

3. S. Enomoto, Boolean algebras and fields of sets, Osaka Math. J. vol. 5 (1953) pp. 99-115.

4. P. Erdös and A. Tarski, On families of mutually exclusive sets, Ann. of Math. vol. 44 (1943) pp. 315-329.

5. A. Horn and A. Tarski, Measures in Boolean algebras, Trans. Amer. Math. Soc. vol. 64 (1948) pp. 467-497. 

(1956).

6. R. S. Pierce, Distributivity in Boolean algebras, Bull. Amer. Math. Soc. Abstract 62-1-44

7. D. Scott, The independence of certain distributive laws in Boolean algebras, Trans. Amer. Math. Soc. vol. 84 (1957) pp. 258-261.

8. R. Sikorski, On the representation of Boolean algebras as fields of sets, Fund. Math. vol. 35 (1948) pp. 247-258.

9. E. C. Smith, Jr., A distributivity condition for Boolean algebras, Ann. of Math. vol. 64 (1956) pp. 551-561.

10. A Tarski, Ideale in vollständigen Mengenkörpern, part I, Fund. Math. vol. 32 (1939) pp. 45-63; part II, Fund. Math. vol. 33 (1945) pp. 51-65.

11. - Über additive und multiplikative Mengenkörpern und Mengenfunktionen, Comptes rendus des Séances de la Société des Sciences et des Lettres de Varsovie, Classe III, vol. 30 (1937) pp. 151-181.

12. - Sur les classes d'ensembles closes par rapport a certaines opérations elémentaires, Fund. Math. vol. 16 (1930) pp. 181-304.

13. - Axiomatic and algebraic aspects of two theorems on sums of cardinals, Fund. Math. vol. 35 (1948) pp. 79-104. 198.

14. —, Zur Grundlegung der Boole'schen algebra, Fund. Math. vol. 24 (1935) pp. 177-

15. - Cardinal algebras, New York, 1949.

16. —- Über unerreichbare Kardinalzahlen, Fund. Math. vol. 30 (1938) pp. 68-89.

17. —_ Drei Überdeckungssätze der allgemeinen Mengenlehre, Fund. Math. vol. 30 (1938) pp. 132-155.

18. S. Ulam, Zur Masstheorie in der allgemeinen Mengenlehre, Fund. Math. vol. 16 (1930) pp. 140-150.

International Business Machines Corp., Los Angeles, Calif.

Brown UNIVERSITY, Providence, R. I.

University of CaLIFornia, Berkeley, Calif. 